\title{
Identification and Localization of Prostate Cancer with Combined Use of T2-Weighted, Diffusion Weighted MRI and Proton MR Spectroscopy, Correlation with Histopathology
}

\author{
Baki Hekimoğlu ${ }^{1}$, Hasan Aydin ${ }^{1}$, Volkan Kızılgöz' ${ }^{1}$ İdil Güneş Tatar ${ }^{1}$, Ali Rıza Ugan² ${ }^{\text {İrem Paker }}{ }^{3}$ \\ ${ }^{1}$ Radiology Department, Dışkapı Yıldırım Beyazıt Education and Research Hospital, Ankara, Turkey \\ ${ }^{2}$ Urology Department, Dışkapı Yıldırım Beyazıt Education and Research Hospital, Ankara, Turkey \\ ${ }^{3}$ Pathology Department, Dışkapı Yıldırım Beyazıt Education and Research Hospital, Ankara, Turkey \\ Email: dr.hasanaydin@hotmail.com
}

Received May 1, 2013; revised June 3, 2013; accepted June 10, 2013

Copyright (C) 2013 Baki Hekimoğlu et al. This is an open access article distributed under the Creative Commons Attribution License, which permits unrestricted use, distribution, and reproduction in any medium, provided the original work is properly cited.

\begin{abstract}
Purpose: To predict the diagnostic performance of combined use of T2-weighted imaging (T2W)-diffusion weighted MRI (DWI) and apparent diffusion coefficient (ADC)-proton MR spectroscopy (H-MRS) for the detection of prostate cancer, correlated to histopathology as the reference standard. Method: After institutional review board approval, 40 patients with prostate cancer were included in this retrospective research. Two readers evaluated the results of T2W, DWI-ADC mapping and H-MRS independently for the depiction of prostate cancer. Reference standard was the TRUSguided biopsy and the surgical histopathological results. Statistical analysis was assessed by Fisher's exact t-test, Wilcoxon signed rank test, variance analysis test with Kappa (k) values and receiver operating characteristics (ROC) curve for $\mathrm{ADC}$ values, Cho/Cit and Cho + Cre/Cit ratios for each observer. Results: Both readers declined $46 \%$ sensitivity and $68 \%$ specificity for T2W sequence, $29 \%$ sensitivity and $82 \%$ specificity for DWI-ADC mapping and $49 \%$ specificity for Cho/Cit and Cho + Cre/Cit ratios, 69\% sensitivity for Cho/Cit 70\% sensitivity for Cho + Cre/Cit ratios of H-MRS. T2W + DWI-ADC mapping + H-MRS (Cho/Cit and Cho + Cre/Cit ratios) regarded 81\% sensitivity and 66\% specificity, with significant statistical differences to the reference histopathology $(\mathrm{p}<0.05)$. Conclusion: Combination of T2W, DWI and H-MRS were more sensitive and more accurate than either sequences alone, for prostate cancer localization and detection.
\end{abstract}

Keywords: MRI; Prostate Cancer; DWI; H-MRS; T2W

\section{Introduction}

For the elder men with high blood level of prostate-specific antigen (PSA), the definite diagnosis of prostate cancer is based on biopsy, obtained invasively by endorectal sonographic guidance (TRUS) [1-3]. However, it has several limitations like, lower sensitivity and specificity for the detection and local staging of prostate cancer, limited potential to delineate the malignant foci, lower diagnostic rate for extra-capsular extension of disease, have high false-negative rates up to $40 \%$ and at the same time high false-positive rates due to benign prostatic disease like prostatitis, hemorrhage, prostate hyperplasia and post-treatment sequela [1,3-5]. MR imaging, a non-invasive diagnostic tool, has shown a great confidence for the evaluation and management of pros- tate cancer [1,2,4-9]. MRI of prostate with combined pelvic and endorectal coil, has become an accepted method for staging of this cancer [1,4,6,9-12], T2W-MR imaging facilitate appropriate data for the treatment planning of prostate cancer via aiding for the detection, localization and staging of prostate cancer which also include the extra-capsular extension and seminal vesicle invasion $[1,4,7,13,14]$. However, T2W imaging has quite good sensitivity but lower specificity for tumor detection and discrimination of cancer from non-malignant tissues, to further improve the sensitivity and mainly the specificity of it; Functional MR imaging techniques like 3D H-MRS, dynamic contrast enhanced MRI and DWIADC mapping have been proposed to be added to the routine prostate MR protocole for the entire diagnosis of 
cancer $[1,2,4,7,10,12,14-16]$. A significant reduction of citrate(Cit) and elevation of choline(Cho), increased Cho/ $\mathrm{Cit}$ and $\mathrm{Cho}+\mathrm{Cre} / \mathrm{Cit}$ ratios have been documented for the diagnosis of prostatic adenocarcinoma in 3D H-MRS, compared to the normal prostate tissue and recently 3D-chemical shift imaging (CSI) has been shown to facilitate sensitivity and specificity for tumor detection up to 95\% respectively [2,4-6,8-11]. DWI-ADC mapping also has potentials for the detection of prostate cancer; ADC values have clinical utility in the depiction of prostate cancer, mean $\mathrm{ADC}$ values for malignant peripheral-transitional and central zone prostatic foci are lower than of those benign prostatic tissues [2,4,7,12,14,16-19]. Addition of those high-MR imaging techniques to routine $\mathrm{T} 2 \mathrm{~W}$ prostate imaging have been found to improve the detection and localization of prostatic cancer $[2,4,7,12,14$, 15,17]. The goal of our present study was to utilize retrospectively the diagnostic performance of combined use of T2W, DWI-ADC mapping and H-MRS for the detection of prostate cancer, correlated to histopathology as the reference standard.

\section{Material and Methods}

The research was approved by institutional review board. Between June 2010 and September 2012, 40 consecutive male patients; age ranged between 54 - 82 years, 69 years mean) with histologically proven prostate cancer without any contraindications for an MR examination of the prostate, were included in this retrospective research. For the analysis of ADC values and $\mathrm{H}-\mathrm{MRS}$ (Cho/Cit and Cho + Cre/Cit ratios) of cancer patients, data of 5 normal control group patients were taken as the reference. None of the patients had previous surgical procedures, radiation or hormonal therapy and none of the patients were excluded from the research due to image distortion. Patients were admitted to MR unit from Urology Department, all the patients underwent a clinical rectal examination, blood PSA levels (total-free) and biopsy yields were also examined. The interval between MRI and TRUS-guided biopsy was 2 - 6 weeks, 4 weeks mean and the surgical approaches were about $1-3$ weeks after MRI. All the MRI procedures and Multi-voxel spectroscopic analysis were carried out with an 8-channel 1.5 T MR scanner (Philips Achieva, Philips Medical systems, Netherlands) by using a 4-channel standard pelvic-phased array coil. Whole prostate and seminal vesicles were visualized in every patient.

Parameters of prostate imaging in this research were, T2W sequence: Performed in axial and coronal planes with turbo spin echo, for axial one; 400*400 FOV, 240*320 matrix, 5175/64: TR/TE, $4 \mathrm{~mm}$ slice thickness, 2 NEX, duration of scan about $2.27 \mathrm{~min}$, for coronal plane; 200*200 FOV, 256*320 matrix, 3800/104: TR/TE, 4 mm slice thickness, 2 NEX, continued about $2.33 \mathrm{~min}$. DWI-
ADC mapping: Performed in transverse plane with 3D-echo planar imaging $300 * 400 \mathrm{FOV}, 144 * 192$ matrix, 2200/84: TR/TE, $2 \mathrm{~mm}$ slice thickness, acquisition time about $2.37 \mathrm{~min}, \mathrm{~b}=800 \mathrm{~s} / \mathrm{mm}^{2}$ ADC values were manually constructed on a pixel-by-pixel basis from six parts of prostate. H-MRS acquisitions: Organized in 3D (Axialsagittal-coronal) planes, Multivoxel approach with pointresolved spectroscopic sequence (PRESS); 200*200 FOV, $1500 / 135$ msec.: TR/TE, time of scan was about 5.45 $\min$.

12 of the patients had several osteoblastic bone metastasis (Dorsal-lumbosacral vertebrae, bilateral iliac wings and sacroiliac joints, ribs and sternoclavicular joints), pelvic-abdominal lymphadenopathies which had been undergone to radiotherapy and chemotherapy, considered as inoperable group, TRUS-guided biopsy was the reference standard in this group. Remaining 28 patients had operation; 5 of them undergone to transurethral resection and 23 had radical prostatectomy, gold standard was the operation and histopathological results in this group. All the surgical procedures were performed by a 15 years experienced urologic surgeon and his team-mates. Analysis of data set: For tumour localization; Prostate was divided into 2 halves: Right (R) and Left (L), then to 3 parts: Apex(A)-transitional(T) zone (middle)-periphe$\mathbf{r a l}(\mathbf{P})$ zone(base), each part then was divided into three compartments (Median-mid and lateral). Thus in each case, we had 18 regions of interest (ROI) within the whole prostate of each patient. In order to avoid discordance of exact localizations for image evaluations and the biopsy site through whole prostate, multiple ROI's were placed through each halves, parts and compartments of each prostate. The readers graded the ROI positive if cancer was found out in any compartment of $R$ and L, A-T and P. Two radiologists (Reader 1 and 2) with 3 and 2 years of experience for the interpretation of $\mathrm{T} 2 \mathrm{~W}$, DWI-ADC mapping and H-MRS respectively, they independently reviewed all images of MRI, spectral analysis and post-processing were carried out by using a software of Philips Achieva Netherland work-shop. Both readers were aware that all MR sequences included in this research, were derived from patients with a biopsy-proven prostate cancer but were unaware of the major cancer localizations of through the prostate histopathologically, also had no prior knowledge about PSA levels and Gleason scores of patients. Both readers presented the H-MRS and ADC results with consensus, for $\mathrm{T} 2 \mathrm{~W}$ sequence; In case of discordance between readers: Reader 3 with 7 years of experience, reviewed the unconsensed images and predicted the final decision. There were no intra-interobserver variability between both readers, no variability observed between reader 3 and the other readers either $(p>0.05)$. Both readers evaluated all the MR images in a random patient case order. At first, 
T2W images were evaluated, the criteria for cancer presence was a mass or nodule homogenously low-signal intensity with ill-defined margins $[1,4,8,10,12]$. Second, DWI-ADC mapping were interpreted, diagnostic criteria was the focal or conglomerated areas or lesions, hyperintense in DWI and hypointense in ADC mapping, relative to surrounding prostate $[1,4,7,12,16-19]$. ADC values of each ROI for both prostate cancer and healthy control group patients were measured manually on the Philips Achieva work station, then compared to each other statistically. ROC curve analysis according to ADC values of both group patients for both readers, were also investigated. Third, H-MRS was evaluated: Multivoxel approach with PRESS voxel excitation by band-selective inversion with gradient dephasing, water-lipid suppression and spatial encoding by chemical shift imaging with high resolution at all three dimensions via 3D-TE: 135 acquisition [4-6,9,20-23]. With the application of Hamming filter; Effective standard voxel size of $1 \times 1 \times 1.5$ $\mathrm{cm}$ was obtained, magnetic field homogeneity was optimized by using both automated and manual shimming, phase encoding was applied to produce 3D-MR spectroscopic arrays of proton spectra throughout the prostate $[2,4,5,7,21,24]$, after post-processing of the time-domain by zero filling to 1024 points, multiplication by Hanning filter, Fourier transformation and phase-baseline correction, spectral data was analyzed to provide standard deviation and peak estimates of choline(Cho), citrate(Cit) and creatine(Cre) resonances, Cit resonance was found at $2.6 \mathrm{ppm}$, Cre at 3.0 and Cho resonance at $3.2 \mathrm{ppm}$, respectively $[4,6,9,21,23,25-28]$. For further analysis, Cho/ $\mathrm{Cit}$ and $\mathrm{Cho}+\mathrm{Cre} / \mathrm{Cit}$ ratios were used for the tumour depiction; Voxels with more than $30 \%$ tumour based on biopsy, were taken as positive ROI for the presence of cancer. Voxels were considered to be malignant if Cho/Cit and $\mathrm{Cho}+\mathrm{Cre} / \mathrm{Cit}$ ratios were at least 2 standard deviations higher than the average ratio of the normal control group results $[2,4,6,8,9,21,25]$. Both ratios for each ROI with prostate cancer and healthy control group patients were measured automatically on the workstation, results of both groups compared to each other statistically, ROC curve analysis according to $\mathrm{Cho} / \mathrm{Cit}$ and $\mathrm{Cho}+\mathrm{Cre} / \mathrm{Cit}$ ratios of both group patients for both readers were also determined.

In order to prevent influences of $\mathrm{T} 2 \mathrm{~W}$ imaging upon later sequences and creating a bias through all three MRI sequences, both readers independently evaluated T2W datas of all patients in a different case order, then analysed the forthcoming sequences also in a random patient case order, quite different than the order of T2W images so we believe that both readers can accurately interpret the real potentials of DWI-ADC mapping and H-MRS to detect exact localization of prostate cancers.

All the statistical analyses were performed by using a software program (SPSS for Windows, SPSS, ChicagoIllinois). Statistical correlation of $\mathrm{T} 2 \mathrm{~W}$ sequence to biopsy and histopathology, was assessed by Fisher's exact t-test, Wilcoxon signed rank test was used to compare malignant tissue with control group for the following variables: $\mathrm{ADC}$ values, $\mathrm{Cho} / \mathrm{Cit}$ and $\mathrm{Cho}+\mathrm{Cre} / \mathrm{Cit}$ ratios, cancerous nodules were paired to normal prostatic tissue of control group patients. Intra and interobserver variability between readers were calculated by Variance analysis test with Kappa $(\mathrm{k})$ values. $\mathrm{k}$ values between $0.80-1.00$ indicated perfect agreement, $\mathrm{k}$ from 0.6 to 0.8 presented high agreement, $\mathrm{k}$ values between 0.4 to 0.6 indicated moderate agreement, $\mathrm{k}$ value 0.2 to 0.4 presented fair agreement, $\mathrm{k}$ value between $0-0.20$ indicated slight agreement $[29,30] . p<0.05$ was considered to indicate a statistically significant difference for both tests. Receiver operating characteristics (ROC) curve was also fitted to obtain cut-off values for $\mathrm{ADC}$, Cho/Cit and Cho + Cre/Cit ratios for each observer to determine malignant prostate nodules by using area under this curve (AUC).

\section{Results}

Tables 1(a) and (b) summarizes the data of patients including, age-PSA levels-Gleason scores, readers results and common lists for T2W and for DWI-ADC values, MRS acquisition of $\mathrm{Cho} / \mathrm{Cit}$ and $\mathrm{Cho}+\mathrm{Cre} / \mathrm{Cit}$ ratios for cancer and normal control group patients. Gleason scores of the patients ranged between $5-10$, mean $3+4=7$, PSA levels of them had the range between $1.6-139.53$ ng/ml, $70.6 \mathrm{ng} / \mathrm{ml}$ : median.

For T2W sequences: Reader 1 declared 100 malignancy and reader 2 predicted 96 cancerous foci through all cancer patients. Both readers presented perfect agreement without any significant statistical differences to each other ( $\mathrm{p}>0.05, \mathrm{k}$ : 0.893). Regarding to biopsy yields; Common results of readers declined $46 \%$ sensitivity and $68 \%$ specificity for the depiction of prostatic cancer with significant statistical differences $(\mathrm{p}<0.05)$ (Table 2, Graphic 1(a), Figure 1).

For DWI and ADC mapping: Reader 1 predicted 50 cancerous areas and reader 2 regarded 43 malignancy through all prostates included in this research. Both readers presented significant statistical differences to each other with perfect agreement ( $p<0.05$, k: 0.900). With regard to biopsy; Common DWI results of readers declined $29 \%$ sensitivity and $82 \%$ specificity for detecting prostate cancer without any statistical differences $(\mathrm{p}$ $>0.05$ ) (Table 2, Figure 2). Mean ADC values in the cancer group was about $5.03 \pm 7.22 \times 10^{-3} \mathrm{~s} / \mathrm{mm}^{2}$, didn't present any significant statistical differences to biopsy results $(\mathrm{p}>0.05), 3.89 \pm 3.69 \times 10^{-3} \mathrm{~s} / \mathrm{mm}^{2}$ in the control group, didn't present any significant statistical differences to biopsy results either ( $p>0.05)$, but both groups 
Table 1. (a) List of patients with results; (b) Control group.

(a)

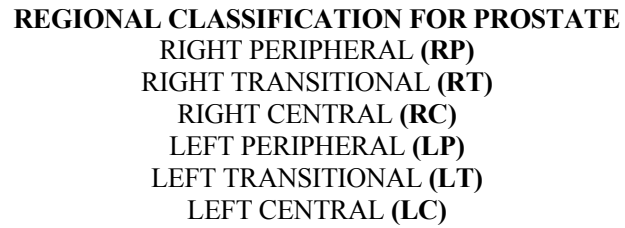

INTEROBSERVER STATEMENT FOR MRS RESULTS DIFFERENCE BETWEEN OBSERVERS:

DBO: Observer $1(+)$, Observer $2(-)$

DBO: Observer $1(-)$, Observer $2(+)$

CON: CONSENSUS ("POSITIVE" ACCORDING TO TWO OBSERVERS AT LEAST)

NN: CONSENSUS ("NEGATIVE" ACCORDING TO TWO OBSERVERS AT LEAST)

IN T1W, T2W, DWI-ADC, C+ COLUMNS RESULTS ARE WRITTEN BY BOLD BLACK LETTERS IF THERE IS NO INTEROBSERVER DIFFERENCE. IN THESE COLUMNS, BLUE LETTERS ARE THE RESULTS REFER ONLY TO OBSERVER 1 AND RED ONES ONLY TO OBSERVER 2

\begin{tabular}{|c|c|c|c|c|c|c|c|c|c|c|c|c|c|}
\hline \multirow{2}{*}{$\mathrm{N}$} & \multirow{2}{*}{ Name } & \multirow{2}{*}{ Age } & \multirow{2}{*}{$\begin{array}{l}\text { tPSA } \\
\text { fPSA }\end{array}$} & \multirow{2}{*}{$\mathrm{T} 2 \mathrm{~W}$} & \multirow{2}{*}{ DWI-ADC } & \multirow{2}{*}{ RATIO } & \multicolumn{6}{|c|}{ MRS } & \multirow{2}{*}{$\begin{array}{c}\text { Biopsy and } \\
\text { Gleason }\end{array}$} \\
\hline & & & & & & & $\mathrm{RP}$ & RT & $\mathrm{RC}$ & LP & LT & $\mathrm{LC}$ & \\
\hline \multirow{2}{*}{1} & \multirow{2}{*}{ A.B. } & \multirow{2}{*}{69} & \multirow{2}{*}{24.67} & \multirow{2}{*}{ RT, RP, LC } & \multirow{2}{*}{ RP, LP } & $\mathrm{Cho} / \mathrm{Ci}$ & $\mathrm{CON}$ & $\mathrm{CON}$ & $\mathrm{CON}$ & $\mathrm{CON}$ & $\mathrm{CON}$ & CON & \multirow{2}{*}{$\begin{array}{c}\mathrm{LC}, \mathrm{RC}, \mathrm{LP} \\
\mathbf{3}+\mathbf{3}=\mathbf{6}\end{array}$} \\
\hline & & & & & & $\mathrm{Cho}+\mathrm{Cr} / \mathrm{CI}$ & $\mathrm{CON}$ & $\mathrm{CON}$ & $\mathrm{CON}$ & $\mathrm{CON}$ & $\mathrm{CON}$ & $\mathrm{CON}$ & \\
\hline \multirow{2}{*}{2} & \multirow{2}{*}{ B.A. } & \multirow{2}{*}{67} & \multirow{2}{*}{45.97} & \multirow{2}{*}{$\begin{array}{l}\text { LP, LT, RP, } \\
\text { RC, LC }\end{array}$} & \multirow{2}{*}{ RC } & $\mathrm{Cho} / \mathrm{Ci}$ & $\mathrm{CON}$ & $\mathrm{CON}$ & $\mathrm{CON}$ & $\mathrm{CON}$ & DBO & $\mathrm{CON}$ & \multirow{2}{*}{$\begin{array}{c}\mathrm{RP}, \mathrm{RT}, \mathrm{RC} \\
\mathrm{LP}, \mathrm{LT}, \mathrm{LC} \\
3+3=6\end{array}$} \\
\hline & & & & & & $\mathrm{Cho}+\mathrm{Cr} / \mathrm{CI}$ & $\mathrm{CON}$ & $\mathrm{CON}$ & $\mathrm{CON}$ & $\mathrm{CON}$ & DBO & $\mathrm{CON}$ & \\
\hline \multirow{2}{*}{3} & \multirow{2}{*}{ C.A. } & \multirow{2}{*}{71} & \multirow{2}{*}{24.13} & \multirow{2}{*}{$\mathbf{R P}, \mathbf{L P}$} & \multirow{2}{*}{$\mathrm{RP}$} & $\mathrm{Cho} / \mathrm{Ci}$ & $\mathrm{CON}$ & $\mathrm{NN}$ & $\mathrm{NN}$ & $\mathrm{NN}$ & $\mathrm{CON}$ & $\mathrm{CON}$ & \multirow{2}{*}{$\begin{array}{c}\mathrm{LP}, \mathrm{LT} \\
\mathbf{3}+\mathbf{3}=\mathbf{6}\end{array}$} \\
\hline & & & & & & $\mathrm{Cho}+\mathrm{Cr} / \mathrm{CI}$ & $\mathrm{CON}$ & $\mathrm{NN}$ & $\mathrm{NN}$ & $\mathrm{NN}$ & $\mathrm{CON}$ & $\mathrm{CON}$ & \\
\hline 4 & $\mathrm{OB}$ & 77 & 22.86 & LP. LC & LP & $\mathrm{Cho} / \mathrm{Ci}$ & $\mathrm{NN}$ & $\mathrm{CON}$ & $\mathrm{CON}$ & $\mathrm{CON}$ & $\mathrm{CON}$ & $\mathrm{CON}$ & LP, LT, LC \\
\hline & & & & & & $\mathrm{Cho}+\mathrm{Cr} / \mathrm{CI}$ & $\mathrm{NN}$ & $\mathrm{CON}$ & $\mathrm{CON}$ & $\mathrm{CON}$ & $\mathrm{CON}$ & $\mathrm{CON}$ & \\
\hline 5 & E.B. & 74 & 19.88 & RP. LP & & $\mathrm{Cho} / \mathrm{Ci}$ & DBO & DBO & $\mathrm{CON}$ & $\mathrm{CON}$ & $\mathrm{CON}$ & $\mathrm{CON}$ & $\mathrm{RP}, \mathrm{RT}, \mathrm{RC}$ \\
\hline & & & & wer & & $\mathrm{Cho}+\mathrm{Cr} / \mathrm{CI}$ & DBO & DBO & $\mathrm{CON}$ & $\mathrm{CON}$ & $\mathrm{CON}$ & $\mathrm{CON}$ & $4+5=9$ \\
\hline 6 & E.E. & 68 & 139.53 & $\mathbf{R P}, \mathrm{LP}$ & $R P, L P$ & & $\mathrm{CON}$ & $\mathrm{NN}$ & $\mathrm{NN}$ & $\mathrm{NN}$ & $\mathrm{NN}$ & $\mathrm{NN}$ & $\begin{array}{c}\mathrm{RP}, \mathrm{RT}, \mathrm{RC}, \\
\mathrm{LP}\end{array}$ \\
\hline & & & & & & $\mathrm{Cho}+\mathrm{Cr} / \mathrm{CI}$ & $\mathrm{CON}$ & $\mathrm{NN}$ & $\mathrm{NN}$ & $\mathrm{NN}$ & $\mathrm{NN}$ & $\mathrm{NN}$ & $5+5=10$ \\
\hline 7 & G.A. & 74 & 7.87 & LP & $\mathbf{N N}$ & $\mathrm{Cho} / \mathrm{Ci}$ & $\mathrm{CON}$ & $\mathrm{CON}$ & $\mathrm{CON}$ & $\mathrm{CON}$ & $\mathrm{CON}$ & $\mathrm{CON}$ & RP, LP \\
\hline & & & & & & $\mathrm{Cho}+\mathrm{Cr} / \mathrm{CI}$ & $\mathrm{CON}$ & $\mathrm{CON}$ & $\mathrm{CON}$ & $\mathrm{CON}$ & $\mathrm{CON}$ & $\mathrm{CON}$ & $4+4=8$ \\
\hline 8 & $\mathrm{H}$ & 69 & 100 & LP IT RT BC & P & $\mathrm{Cho} / \mathrm{Ci}$ & $\mathrm{NN}$ & $\mathrm{CON}$ & $\mathrm{NN}$ & $\mathrm{CON}$ & $\mathrm{CON}$ & $\mathrm{CON}$ & LC, LP \\
\hline & ก.ก. & (5) & 16.4 & $\mathbf{L I}, \mathbf{L I}, \mathbf{N}, \mathbf{L}$ & 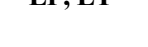 & $\mathrm{Cho}+\mathrm{Cr} / \mathrm{CI}$ & $\mathrm{NN}$ & $\mathrm{CON}$ & $\mathrm{NN}$ & $\mathrm{CON}$ & $\mathrm{CON}$ & $\mathrm{CON}$ & $4+4=8$ \\
\hline 9 & H.Ü. & 64 & 10.97 & RT, RP, LP & & $\mathrm{Cho} / \mathrm{Ci}$ & $\mathrm{CON}$ & $\mathrm{CON}$ & $\mathrm{CON}$ & $\mathrm{CON}$ & $\mathrm{CON}$ & $\mathrm{NN}$ & LP, LT, LC \\
\hline & & & & & & $\mathrm{Cho}+\mathrm{Cr} / \mathrm{CI}$ & $\mathrm{CON}$ & $\mathrm{CON}$ & $\mathrm{CON}$ & $\mathrm{CON}$ & $\mathrm{CON}$ & $\mathrm{NN}$ & \\
\hline $\mathrm{N}$ & Name & Age & tPSA & T2W & DWI-ADC & RATIO & & & & & & & Biopsy and \\
\hline & & & fPSA & & & & $\mathrm{RP}$ & RT & $\mathrm{RC}$ & LP & LT & $\mathrm{LC}$ & Gleason \\
\hline 10 & HY & 78 & 8.01 & JP $\mathrm{B}$ & PP & $\mathrm{Cho} / \mathrm{Ci}$ & $\mathrm{CON}$ & $\mathrm{NN}$ & $\mathrm{CON}$ & $\mathrm{CON}$ & $\mathrm{CON}$ & $\mathrm{NN}$ & RP, RT, RC, \\
\hline & & & 10.2 & & & $\mathrm{Cho}+\mathrm{Cr} / \mathrm{CI}$ & $\mathrm{CON}$ & $\mathrm{NN}$ & $\mathrm{CON}$ & $\mathrm{CON}$ & $\mathrm{CON}$ & $\mathrm{NN}$ & $3+3=6$ \\
\hline
\end{tabular}

MR SEQUENCES

T2 WEIGHTED (T2W)

DIFFUSION AND ADC (DWI-ADC)

MR SPECTROSCOPY (MRS)

PSA: Prostate Spesific Antigen $(\mathrm{ng} / \mathrm{mL})$

fPSA: Free PSA level, tPSA: Total PSA level

IN THE LAST COLUMN, "BIOPSY" MEANS BIOPSY RESULTS. THIS COLUMN INDICATES THE REGIONS WHICH ARE MALIGNANT ACCORDING TO PATOLOGY RESULT AND “GLEASON" MEANS THE GLEASON SCORE OF THE MALIGNANT REGIONS

THE GREEN LINES INDICATE THE PATIENTS IN CONTROL GROUP

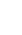


Continued

\begin{tabular}{|c|c|c|c|c|c|c|c|c|c|c|c|c|c|}
\hline \multirow{2}{*}{11} & \multirow{2}{*}{ H.K. } & \multirow{2}{*}{80} & \multirow{2}{*}{59.26} & \multirow{2}{*}{ LP, RP, LT } & \multirow{2}{*}{ LP, LT } & $\mathrm{Cho} / \mathrm{Ci}$ & $\mathrm{CON}$ & $\mathrm{CON}$ & $\mathrm{NN}$ & $\mathrm{CON}$ & $\mathrm{CON}$ & $\mathrm{NN}$ & \multirow{2}{*}{$\begin{array}{c}\text { RP, RT, RC, } \\
\text { LP, LT, LC } \\
4+4=8\end{array}$} \\
\hline & & & & & & $\mathrm{Cho}+\mathrm{Cr} / \mathrm{CI}$ & $\mathrm{CON}$ & $\mathrm{CON}$ & $\mathrm{NN}$ & $\mathrm{CON}$ & $\mathrm{CON}$ & $\mathrm{NN}$ & \\
\hline \multirow{2}{*}{12} & \multirow{2}{*}{ R.Ç. } & \multirow{2}{*}{68} & \multirow{2}{*}{10.6} & \multirow{2}{*}{ RP, RT, LP, LT } & \multirow{2}{*}{ LP, RP } & $\mathrm{Cho} / \mathrm{Ci}$ & $\mathrm{CON}$ & $\mathrm{CON}$ & $\mathrm{CON}$ & $\mathrm{CON}$ & $\mathrm{NN}$ & $\mathrm{CON}$ & \multirow{2}{*}{$\begin{array}{c}\mathrm{RP}, \mathrm{RT}, \mathrm{RC} \\
\mathbf{4}+\mathbf{4}=\mathbf{8}\end{array}$} \\
\hline & & & & & & $\mathrm{Cho}+\mathrm{Cr} / \mathrm{CI}$ & $\mathrm{CON}$ & $\mathrm{CON}$ & $\mathrm{CON}$ & $\mathrm{CON}$ & $\mathrm{NN}$ & $\mathrm{CON}$ & \\
\hline \multirow{2}{*}{13} & \multirow{2}{*}{ H.S. } & \multirow{2}{*}{74} & \multirow{2}{*}{5.28} & \multirow{2}{*}{ NN } & \multirow{2}{*}{$\mathrm{RP}$} & $\mathrm{Cho} / \mathrm{Ci}$ & $\mathrm{CON}$ & $\mathrm{NN}$ & $\mathrm{NN}$ & $\mathrm{NN}$ & $\mathrm{CON}$ & $\mathrm{NN}$ & \multirow{2}{*}{$\begin{array}{c}\mathrm{LT} \\
\mathbf{3}+\mathbf{3}=\mathbf{6}\end{array}$} \\
\hline & & & & & & $\mathrm{Cho}+\mathrm{Cr} / \mathrm{CI}$ & $\mathrm{CON}$ & $\mathrm{NN}$ & $\mathrm{NN}$ & $\mathrm{NN}$ & $\mathrm{CON}$ & $\mathrm{NN}$ & \\
\hline \multirow{2}{*}{14} & İ.A & 77 & 31.02 & RP. & JP & $\mathrm{Cho} / \mathrm{Ci}$ & $\mathrm{CON}$ & $\mathrm{CON}$ & $\mathrm{NN}$ & $\mathrm{NN}$ & $\mathrm{NN}$ & $\mathrm{CON}$ & $\mathrm{RT}, \mathrm{RC}, \mathrm{LT}$, \\
\hline & & & 3.89 & M & Li & $\mathrm{Cho}+\mathrm{Cr} / \mathrm{CI}$ & $\mathrm{CON}$ & $\mathrm{CON}$ & $\mathrm{NN}$ & $\mathrm{NN}$ & $\mathrm{NN}$ & $\mathrm{CON}$ & $4+3=7$ \\
\hline 15 & İU & 81 & 70.14 & RT & $\mathbf{N N}$ & $\mathrm{Cho} / \mathrm{Ci}$ & $\mathrm{NN}$ & $\mathrm{NN}$ & $\mathrm{CON}$ & $\mathrm{CON}$ & $\mathrm{CON}$ & $\mathrm{CON}$ & $\mathrm{RP}, \mathrm{RT}, \mathrm{RC}$, \\
\hline & & & & & & $\mathrm{Cho}+\mathrm{Cr} / \mathrm{CI}$ & $\mathrm{NN}$ & $\mathrm{CON}$ & $\mathrm{CON}$ & $\mathrm{CON}$ & $\mathrm{CON}$ & $\mathrm{CON}$ & $3+3=6$ \\
\hline 16 & $\dot{I} \mathrm{~V}$ & 65 & $120 ?$ & DT & $\mathrm{PD}$ & $\mathrm{Cho} / \mathrm{Ci}$ & DBO & $\mathrm{NN}$ & NN & $\mathrm{NN}$ & $\mathrm{NN}$ & $\mathrm{CON}$ & $\mathrm{RC}$ \\
\hline 10 & 1.1. & $0 J$ & 12.02 & $\mathrm{NT}, \mathrm{NI}$ & K, & $\mathrm{Cho}+\mathrm{Cr} / \mathrm{CI}$ & DBO & $\mathrm{NN}$ & $\mathrm{NN}$ & $\mathrm{NN}$ & $\mathrm{NN}$ & $\mathrm{CON}$ & $2+3=5$ \\
\hline 17 & A $S$ & 71 & 4573 & LC. RC IP RT & LP & $\mathrm{Cho} / \mathrm{Ci}$ & $\mathrm{NN}$ & $\mathrm{NN}$ & $\mathrm{CON}$ & $\mathrm{NN}$ & $\mathrm{NN}$ & $\mathrm{CON}$ & $\mathrm{RP}, \mathrm{RT}, \mathrm{RC}$, \\
\hline & & & & & & $\mathrm{Cho}+\mathrm{Cr} / \mathrm{CI}$ & $\mathrm{NN}$ & $\mathrm{NN}$ & $\mathrm{CON}$ & $\mathrm{NN}$ & $\mathrm{NN}$ & CON & $3+4=7$ \\
\hline 18 & MS. & 62 & 26.40 & RP. LP & RP & $\mathrm{Cho} / \mathrm{Ci}$ & $\mathrm{CON}$ & $\mathrm{CON}$ & $\mathrm{CON}$ & $\mathrm{CON}$ & $\mathrm{CON}$ & $\mathrm{CON}$ & $\mathrm{RP}, \mathrm{RT}, \mathrm{RC}$ \\
\hline & & & & W & & $\mathrm{Cho}+\mathrm{Cr} / \mathrm{CI}$ & $\mathrm{CON}$ & $\mathrm{CON}$ & $\mathrm{CON}$ & $\mathrm{CON}$ & $\mathrm{CON}$ & $\mathrm{CON}$ & $3+3=6$ \\
\hline $\mathrm{N}$ & Name & Age & tPSA & T2W & DWI_ADC & RATIO & & & M & & & & Biopsy and \\
\hline 10 & Nante & Age & fPSA & $12 \mathrm{w}$ & DVI-ADC & NATIU & $\mathrm{RP}$ & RT & $\mathrm{RC}$ & LP & LT & $\mathrm{LC}$ & Gleason \\
\hline 19 & H.K. & 68 & 6.35 & RP, RT, LP, LC & RP, RT & $\mathrm{Cho} / \mathrm{Ci}$ & $\mathrm{CON}$ & $\mathrm{NN}$ & $\mathrm{NN}$ & $\mathrm{CON}$ & NN & $\mathrm{NN}$ & LP \\
\hline & & & & & & $\mathrm{Cho}+\mathrm{Cr} / \mathrm{CI}$ & $\mathrm{CON}$ & $\mathrm{NN}$ & $\mathrm{NN}$ & $\mathrm{CON}$ & $\mathrm{NN}$ & $\mathrm{NN}$ & $+3=6$ \\
\hline 20 & MY & 68 & 18.42 & LP LT LC RP & LP LT LC RP & $\mathrm{Cho} / \mathrm{Ci}$ & $\mathrm{CON}$ & $\mathrm{CON}$ & $\mathrm{CON}$ & $\mathrm{CON}$ & $\mathrm{CON}$ & $\mathrm{CON}$ & LP, LT, LC \\
\hline & & & & {$\left[\begin{array}{ll}\mathrm{L} \\
\mathrm{H}\end{array}\right.$} & {$[1,+1, \ldots, \ldots$} & $\mathrm{Cho}+\mathrm{Cr} / \mathrm{CI}$ & $\mathrm{CON}$ & $\mathrm{CON}$ & $\mathrm{CON}$ & $\mathrm{CON}$ & $\mathrm{CON}$ & $\mathrm{CON}$ & $3+4=7$ \\
\hline 21 & $M G$ & 72 & 11.19 & RP. LP & RP. LP & $\mathrm{Cho} / \mathrm{Ci}$ & DBO & $\mathrm{NN}$ & $\mathrm{CON}$ & $\mathrm{NN}$ & $\mathrm{CON}$ & $\mathrm{CON}$ & LP, LT, LC, \\
\hline & & & 1.33 & & & $\mathrm{Cho}+\mathrm{Cr} / \mathrm{CI}$ & DBO & $\mathrm{NN}$ & $\mathrm{CON}$ & NN & $\mathrm{CON}$ & CON & $4+4=8$ \\
\hline 22 & M.K. & 60 & 37.39 & RP & RP & $\mathrm{Cho} / \mathrm{Ci}$ & $\mathrm{CON}$ & $\mathrm{CON}$ & $\mathrm{CON}$ & $\mathrm{CON}$ & $\mathrm{NN}$ & $\mathrm{NN}$ & $\mathrm{RP}, \mathrm{RT}$ \\
\hline & & & & & & $\mathrm{Cho}+\mathrm{Cr} / \mathrm{CI}$ & $\mathrm{CON}$ & $\mathrm{CON}$ & $\mathrm{CON}$ & $\mathrm{CON}$ & $\mathrm{NN}$ & $\mathrm{NN}$ & $3+3=6$ \\
\hline 23 & $H_{G}$ & 73 & 8562 & RP LP LC RT & & $\mathrm{Cho} / \mathrm{Ci}$ & $\mathrm{CON}$ & $\mathrm{NN}$ & $\mathrm{NN}$ & $\mathrm{CON}$ & $\mathrm{NN}$ & $\mathrm{NN}$ & RP, RT, RC, \\
\hline & & & & & & $\mathrm{Cho}+\mathrm{Cr} / \mathrm{CI}$ & $\mathrm{CON}$ & $\mathrm{NN}$ & NN & $\mathrm{CON}$ & $\mathrm{NN}$ & $\mathrm{NN}$ & $3+4=7$ \\
\hline 24 & ÖK & 62 & 747 & LP RT I $\mathrm{RC}$ & $R$ & $\mathrm{Cho} / \mathrm{Ci}$ & $\mathrm{CON}$ & $\mathrm{NN}$ & $\mathrm{CON}$ & $\mathrm{CON}$ & $\mathrm{NN}$ & $\mathrm{CON}$ & $\mathrm{RP}, \mathrm{RT}$ \\
\hline 27 & W. & 02 & $1.7 /$ & $L 1, N, L C, N$ & Le, $\mathrm{K}$ & $\mathrm{Cho}+\mathrm{Cr} / \mathrm{CI}$ & $\mathrm{CON}$ & $\mathrm{NN}$ & $\mathrm{CON}$ & $\mathrm{CON}$ & $\mathrm{NN}$ & $\mathrm{CON}$ & $3+3=6$ \\
\hline 25 & ÖK & 78 & 7229 & RP RT I T & RP RT & $\mathrm{Cho} / \mathrm{Ci}$ & $\mathrm{CON}$ & $\mathrm{CON}$ & NN & $\mathrm{NN}$ & $\mathrm{CON}$ & $\mathrm{NN}$ & RP, RT, RC, \\
\hline & & & & & & $\mathrm{Cho}+\mathrm{Cr} / \mathrm{CI}$ & $\mathrm{CON}$ & $\mathrm{CON}$ & $\mathrm{NN}$ & $\mathrm{NN}$ & $\mathrm{CON}$ & $\mathrm{NN}$ & $4+4=8$ \\
\hline 26 & R P & 71 & 18.51 & & 1 & $\mathrm{Cho} / \mathrm{Ci}$ & $\mathrm{CON}$ & $\mathrm{NN}$ & $\mathrm{NN}$ & $\mathrm{NN}$ & $\mathrm{CON}$ & $\mathrm{NN}$ & $\mathrm{RP}, \mathrm{LT}$ \\
\hline & & & 6.93 & & $\mathbf{L F}, \mathbf{L I}$ & $\mathrm{Cho}+\mathrm{Cr} / \mathrm{CI}$ & $\mathrm{CON}$ & $\mathrm{NN}$ & $\mathrm{NN}$ & $\mathrm{NN}$ & $\mathrm{CON}$ & $\mathrm{NN}$ & $3+3=6$ \\
\hline 27 & 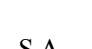 & 75 & 3710 & DP ID DT IT & T $\mathrm{TC}$ & $\mathrm{Cho} / \mathrm{Ci}$ & $\mathrm{CON}$ & $\mathrm{CON}$ & $\mathrm{CON}$ & $\mathrm{CON}$ & $\mathrm{CON}$ & $\mathrm{CON}$ & RP, RT, RC, \\
\hline & & & & & $\mathrm{LI}, \mathrm{NC}, \mathrm{LC}$ & $\mathrm{Cho}+\mathrm{Cr} / \mathrm{CI}$ & $\mathrm{CON}$ & $\mathrm{CON}$ & $\mathrm{CON}$ & $\mathrm{CON}$ & $\mathrm{CON}$ & $\mathrm{CON}$ & $5+4=9$ \\
\hline $\mathrm{N}$ & Name & Age & tPSA & T2W & DWI_ADC & RATIO & & & M & & & & Biopsy and \\
\hline & & & fPSA & & & & $\mathrm{RP}$ & $\mathrm{RT}$ & $\mathrm{RC}$ & LP & LT & $\mathrm{LC}$ & Gleason \\
\hline 28 & $\mathrm{SH}$ & 61 & 20,49 & PP $\mathrm{P}^{\prime}$ & & $\mathrm{Cho} / \mathrm{Ci}$ & $\mathrm{CON}$ & $\mathrm{CON}$ & $\mathrm{CON}$ & $\mathrm{CON}$ & $\mathrm{CON}$ & CON & RP, RT, RC, \\
\hline 20 & 5.11. & 01 & 3.62 & $\mathbf{N}, \mathbf{n}$ & & $\mathrm{Cho}+\mathrm{Cr} / \mathrm{CI}$ & $\mathrm{CON}$ & $\mathrm{CON}$ & $\mathrm{CON}$ & $\mathrm{CON}$ & $\mathrm{CON}$ & $\mathrm{CON}$ & $4+4=8$ \\
\hline
\end{tabular}


Continued

\begin{tabular}{|c|c|c|c|c|c|c|c|c|c|c|c|c|c|}
\hline \multirow{2}{*}{29} & \multirow{2}{*}{ S.Ö. } & \multirow{2}{*}{78} & \multirow{2}{*}{$\begin{array}{c}10.93 \\
\mathbf{1 . 6 2}\end{array}$} & \multirow{2}{*}{ RP, LP, RT } & \multirow{2}{*}{$\mathbf{R P}$} & $\mathrm{Cho} / \mathrm{Ci}$ & CON & $\mathrm{CON}$ & $\mathrm{NN}$ & $\mathrm{NN}$ & $\mathrm{NN}$ & NN & \multirow{2}{*}{$\begin{array}{c}\mathrm{RP}, \mathrm{RT}, \mathrm{RC} \\
\mathbf{3}+\mathbf{3}=\mathbf{6}\end{array}$} \\
\hline & & & & & & $\mathrm{Cho}+\mathrm{Cr} / \mathrm{CI}$ & CON & $\mathrm{CON}$ & $\mathrm{NN}$ & $\mathrm{NN}$ & $\mathrm{NN}$ & $\mathrm{NN}$ & \\
\hline \multirow{2}{*}{30} & \multirow{2}{*}{ Ş.M.E } & \multirow{2}{*}{82} & \multirow{2}{*}{$\begin{array}{c}15,08 \\
\mathbf{1 . 5 8}\end{array}$} & \multirow{2}{*}{ RP, RT, LP } & \multirow{2}{*}{ RP, RT } & $\mathrm{Cho} / \mathrm{Ci}$ & $\mathrm{CON}$ & $\mathrm{CON}$ & $\mathrm{NN}$ & $\mathrm{CON}$ & $\mathrm{CON}$ & $\mathrm{CON}$ & \multirow{2}{*}{$\begin{array}{c}\text { RP, RT, RC, } \\
\text { LP, LT, LC } \\
\mathbf{3}+\mathbf{5}=\mathbf{8}\end{array}$} \\
\hline & & & & & & $\mathrm{Cho}+\mathrm{Cr} / \mathrm{CI}$ & $\mathrm{CON}$ & $\mathrm{CON}$ & $\mathrm{NN}$ & $\mathrm{CON}$ & $\mathrm{CON}$ & $\mathrm{CON}$ & \\
\hline \multirow{2}{*}{31} & \multirow{2}{*}{ S.Ö. } & \multirow{2}{*}{69} & \multirow{2}{*}{$\begin{array}{l}67.4 \\
19.9\end{array}$} & \multirow{2}{*}{ LP, RP, LT } & \multirow{2}{*}{ LP, RP } & $\mathrm{Cho} / \mathrm{Ci}$ & $\mathrm{CON}$ & $\mathrm{CON}$ & $\mathrm{CON}$ & $\mathrm{CON}$ & $\mathrm{CON}$ & DBO & \multirow{2}{*}{$\begin{array}{c}\mathrm{LT}, \mathrm{RP}, \mathrm{RT} \\
\mathrm{RC} \\
3+3=6 \\
3+4=7\end{array}$} \\
\hline & & & & & & $\mathrm{Cho}+\mathrm{Cr} / \mathrm{CI}$ & $\mathrm{CON}$ & $\mathrm{CON}$ & $\mathrm{CON}$ & $\mathrm{CON}$ & $\mathrm{CON}$ & DBO & \\
\hline \multirow{2}{*}{32} & \multirow{2}{*}{ Z.K. } & 59 & 1657 & LP I T & JP & $\mathrm{Cho} / \mathrm{Ci}$ & $\mathrm{CON}$ & $\mathrm{CON}$ & $\mathrm{CON}$ & $\mathrm{CON}$ & $\mathrm{CON}$ & $\mathrm{CON}$ & LP, RP, RC, \\
\hline & & & & & & $\mathrm{Cho}+\mathrm{Cr} / \mathrm{CI}$ & $\mathrm{CON}$ & $\mathrm{CON}$ & $\mathrm{CON}$ & $\mathrm{CON}$ & CON & $\mathrm{CON}$ & $4+3=7$ \\
\hline 33 & $\Delta \mathrm{K}$ & 70 & 100 & PD PT JP T T & IP PD & $\mathrm{Cho} / \mathrm{Ci}$ & $\mathrm{CON}$ & $\mathrm{CON}$ & $\mathrm{CON}$ & $\mathrm{CON}$ & $\mathrm{CON}$ & $\mathrm{NN}$ & LP, LT, LC, \\
\hline Jכ & A.N. & 10 & 21.17 & $\mathrm{KT}, \mathrm{K}, \mathrm{LT}, \mathrm{LI}$ & $\mathbf{L F}, \mathbf{K}$ & $\mathrm{Cho}+\mathrm{Cr} / \mathrm{CI}$ & $\mathrm{CON}$ & $\mathrm{CON}$ & $\mathrm{CON}$ & $\mathrm{CON}$ & $\mathrm{CON}$ & $\mathrm{NN}$ & $3+4=7$ \\
\hline 34 & MB. & 61 & 4.81 & RP $L P, R C$ & IP I T & $\mathrm{Cho} / \mathrm{Ci}$ & CON & NN & $\mathrm{NN}$ & $\mathrm{CON}$ & $\mathrm{CON}$ & $\mathrm{CON}$ & LP, LT, LC \\
\hline & NII.D. & & 1.05 & M, & $\mathbf{L I}, \mathbf{L 1}$ & $\mathrm{Cho}+\mathrm{Cr} / \mathrm{CI}$ & $\mathrm{CON}$ & $\mathrm{NN}$ & $\mathrm{NN}$ & $\mathrm{CON}$ & $\mathrm{CON}$ & $\mathrm{CON}$ & $3+3=6$ \\
\hline 35 & VÖ & 64 & 56.53 & RP LP RT & $\mathbf{L T}$ & & & & & & & & RP, RT, RC, \\
\hline & & & & & & $\mathrm{Cho}+\mathrm{Cr} / \mathrm{CI}$ & & & & & & & $3+4=7$ \\
\hline 36 & $O F$ & 64 & 1.60 & J I T & & $\mathrm{Cho} / \mathrm{Ci}$ & & & & & & & $\mathrm{RP}, \mathrm{RT}, \mathrm{RC}$, \\
\hline 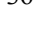 & W.L. & $0 T$ & 0.68 & 2,51 & & $\mathrm{Cho}+\mathrm{Cr} / \mathrm{CI}$ & & & & & & & $3+4=7$ \\
\hline$N$ & Name & Are & tPSA & T2W & DWE $A \mathrm{DC}$ & PATO & & & & & & & Biopsy and \\
\hline 1 & गिपाता & Me & fPSA & $12 \mathrm{~m}$ & (1) & से & $\mathrm{RP}$ & RT & $\mathrm{RC}$ & LP & LT & LC & Gleason \\
\hline 37 & $\mathrm{HC}$ & 50 & 1263 & PP JP & & $\mathrm{Cho} / \mathrm{Ci}$ & & & & & & & LP, RP, RC, \\
\hline Ji & 11.ç & (37 & 12.00 & $\mathrm{NO}, \mathrm{EI}$ & & $\mathrm{Cho}+\mathrm{Cr} / \mathrm{CI}$ & & & & & & & $3+4=7$ \\
\hline 38 & $\mathrm{NA}$ & 62 & 590 & PP IP RT IT & & $\mathrm{Cho} / \mathrm{Ci}$ & & & & & & & LP, LT, LC \\
\hline & & & & & & $\mathrm{Cho}+\mathrm{Cr} / \mathrm{CI}$ & & & & & & & $3+4=7$ \\
\hline 20 & $M$ & 66 & $106-8$ & & & $\mathrm{Cho} / \mathrm{Ci}$ & $\mathrm{NN}$ & $\mathrm{CON}$ & $\mathrm{NN}$ & $\mathrm{NN}$ & $\mathrm{NN}$ & $\mathrm{CON}$ & $\mathrm{RP}, \mathrm{RC}$ \\
\hline 37 & NI.A & 00 & 10.0 & & & $\mathrm{Cho}+\mathrm{Cr} / \mathrm{CI}$ & $\mathrm{NN}$ & $\mathrm{CON}$ & $\mathrm{NN}$ & $\mathrm{NN}$ & $\mathrm{NN}$ & $\mathrm{CON}$ & $3+3=6$ \\
\hline 10 & $M A$ & 51 & 20 & & $\mathbf{P D T}_{\mathrm{T}}$ & $\mathrm{Cho} / \mathrm{Ci}$ & $\mathrm{NN}$ & $\mathrm{NN}$ & $\mathrm{NN}$ & $\mathrm{CON}$ & $\mathrm{CON}$ & $\mathrm{NN}$ & RP, RT, RC \\
\hline 40 & MI.A & 34 & 32.0 & & LF, LI & $\mathrm{Cho}+\mathrm{Cr} / \mathrm{CI}$ & $\mathrm{NN}$ & $\mathrm{NN}$ & $\mathrm{NN}$ & $\mathrm{CON}$ & $\mathrm{CON}$ & $\mathrm{NN}$ & $4+3=7$ \\
\hline & & & & & & (b) & & & & & & & \\
\hline$N$ & Nome & $\Delta 0$ & tPSA & T2W & DUI ADC & PАTИ & & & & & & & Biopsy and \\
\hline & & & fPSA & & & & RP & RT & $\mathrm{RC}$ & LP & LT & LC & Gleason \\
\hline 01 & $\mathrm{HK}$ & 59 & 458 & $\mathbf{T} \mathbf{T}$ & J I T I C & $\mathrm{Cho} / \mathrm{Ci}$ & $\mathrm{NN}$ & $\mathrm{CON}$ & $\mathrm{NN}$ & $\mathrm{CON}$ & $\mathrm{CON}$ & $\mathrm{CON}$ & 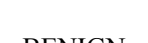 \\
\hline & & & & $\mathbf{H}, \mathbf{E}$ & $\mathrm{EI}, \mathrm{LI}, \mathrm{LC}$ & $\mathrm{Cho}+\mathrm{Cr} / \mathrm{CI}$ & $\mathrm{NN}$ & $\mathrm{CON}$ & $\mathrm{NN}$ & $\mathrm{CON}$ & $\mathrm{CON}$ & $\mathrm{CON}$ & DEFIVIN \\
\hline & & & & & & $\mathrm{Cho} / \mathrm{Ci}$ & $\mathrm{NN}$ & $\mathrm{NN}$ & $\mathrm{CON}$ & $\mathrm{NN}$ & $\mathrm{NN}$ & $\mathrm{NN}$ & \\
\hline 02 & N.A. & 63 & 12.35 & $\mathbf{L P}$ & NN & $\mathrm{Cho}+\mathrm{Cr} / \mathrm{CI}$ & $\mathrm{NN}$ & $\mathrm{NN}$ & $\mathrm{CON}$ & $\mathrm{NN}$ & $\mathrm{NN}$ & $\mathrm{NN}$ & DENIUN \\
\hline & & & & & & $\mathrm{Cho} / \mathrm{Ci}$ & NN & $\mathrm{CON}$ & $\mathrm{NN}$ & $\mathrm{CON}$ & $\mathrm{NN}$ & $\mathrm{NN}$ & \\
\hline 03 & N.T. & 61 & 5.79 & RP, LP, LT & RP, LP & $\mathrm{Cho}+\mathrm{Cr} / \mathrm{CI}$ & $\mathrm{NN}$ & $\mathrm{CON}$ & $\mathrm{NN}$ & $\mathrm{CON}$ & $\mathrm{NN}$ & $\mathrm{NN}$ & BENIGN \\
\hline & $M C$ & 75 & $80 ?$ & $\mathrm{DPC} \mathrm{C}$ & 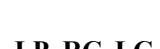 & $\mathrm{Cho} / \mathrm{Ci}$ & $\mathrm{NN}$ & $\mathrm{CON}$ & $\mathrm{NN}$ & $\mathrm{NN}$ & $\mathrm{NN}$ & $\mathrm{NN}$ & \\
\hline 07 & 101.0. & 15 & $0.0 J$ & Li, Ne, Le & $\mathrm{LI}, \mathrm{K}, \mathrm{LC}$ & $\mathrm{Cho}+\mathrm{Cr} / \mathrm{CI}$ & $\mathrm{NN}$ & $\mathrm{CON}$ & $\mathrm{NN}$ & $\mathrm{NN}$ & $\mathrm{NN}$ & $\mathrm{NN}$ & DENIUIN \\
\hline $0=$ & $V$ & 72 & 1 & & & $\mathrm{Cho} / \mathrm{Ci}$ & $\mathrm{NN}$ & $\mathrm{NN}$ & $\mathrm{NN}$ & $\mathrm{NN}$ & $\mathrm{NN}$ & $\mathrm{NN}$ & \\
\hline 00 & К.A. & 12 & 4.90 & & & $\mathrm{Cho}+\mathrm{Cr} / \mathrm{CI}$ & $\mathrm{NN}$ & $\mathrm{NN}$ & $\mathrm{NN}$ & $\mathrm{NN}$ & $\mathrm{NN}$ & $\mathrm{NN}$ & BENIUN \\
\hline
\end{tabular}


Table 2. The results of T2W MRI and DWI-ADC MRI with respect to biopsy results.

\begin{tabular}{|c|c|c|c|c|c|}
\hline & & & \multicolumn{2}{|c|}{ Biopsy } & \multirow{2}{*}{ Total } \\
\hline & & & Positive & Negative & \\
\hline \multirow{9}{*}{$\mathrm{T} 2 \mathrm{~W}$} & \multirow{2}{*}{ Positive } & $\mathrm{n}$ & 67 & 34 & 101 \\
\hline & & $\%$ & $66.3 \%$ & $33.7 \%$ & $100.0 \%$ \\
\hline & \multirow{2}{*}{ Negative } & $\mathrm{n}$ & 80 & 71 & 151 \\
\hline & & $\%$ & $53.0 \%$ & $47.0 \%$ & $100.0 \%$ \\
\hline & \multirow{2}{*}{ Total } & $\mathrm{n}$ & 147 & 147 & 252 \\
\hline & & $\%$ & $58.3 \%$ & $58.3 \%$ & $100.0 \%$ \\
\hline & \multicolumn{4}{|c|}{$\mathrm{p}=0.038 ;$ Sensitivity $=0.46 ;$ Specificity $=0.68$} & \\
\hline & & & \multicolumn{2}{|c|}{ Biopsy } & \\
\hline & & & Positive & Negative & \\
\hline \multirow{6}{*}{$\begin{array}{l}\text { DWI- } \\
\text { ADC }\end{array}$} & & $\mathrm{n}$ & 34 & 17 & 51 \\
\hline & rositive & $\%$ & $66.7 \%$ & $33.3 \%$ & $100.0 \%$ \\
\hline & \multirow{2}{*}{ Negative } & $\mathrm{n}$ & 82 & 77 & 159 \\
\hline & & $\%$ & $51.6 \%$ & $48.4 \%$ & $100.0 \%$ \\
\hline & \multirow{2}{*}{ Total } & $\mathrm{n}$ & 126 & 116 & 210 \\
\hline & & $\%$ & $60.0 \%$ & $55.2 \%$ & $100.0 \%$ \\
\hline \multicolumn{6}{|c|}{$\mathrm{p}=0.075 ;$ Sensitivity $=0.29 ;$ Spesivity $=0.82$} \\
\hline
\end{tabular}

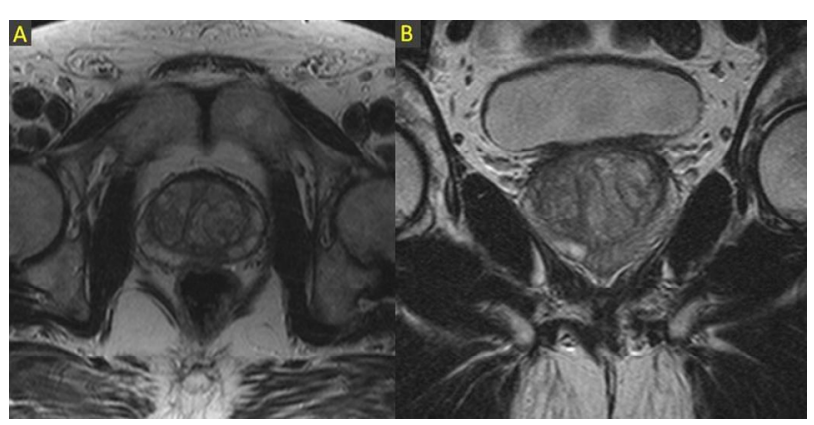

Figure 1. Axial (A) and coronal (B) T2W of a patient. Focal hypointensity in both peripheral regions, especially on the left side.

regarded significant statistical differences to each other according to mean ADC values $(\mathrm{p}<0.05$ ) (Table 3, Graphic 1(a)). With correspondence to ADC values in the cancer group; Threshold cut-off value for the ROC curve was $0.55 \mathrm{~s} / \mathrm{mm}^{2}$, AUC; $0.462 \pm 0.052$. Cut-off values $\geq 0.55 \mathrm{~s} / \mathrm{mm}^{2}$ had $100 \%$ sensitivity and specificity to diagnose prostate cancer with ADC values of cancer patients (Graphic 1b). For the control group; ROC curve regarded cut-off value $0.06 \mathrm{~s} / \mathrm{mm}^{2}$, AUC; $0.389 \pm 0.183$. Cut-off values equal or greater than $0.06 \mathrm{~s} / \mathrm{mm}^{2}$, presented 100\% sensitivity and specificity (Graphic 1(c)).

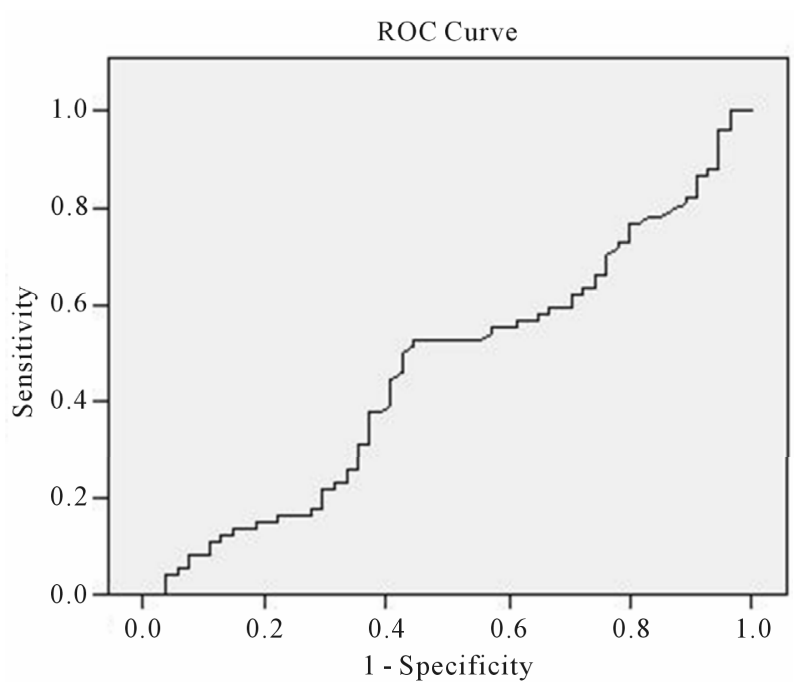

(a)

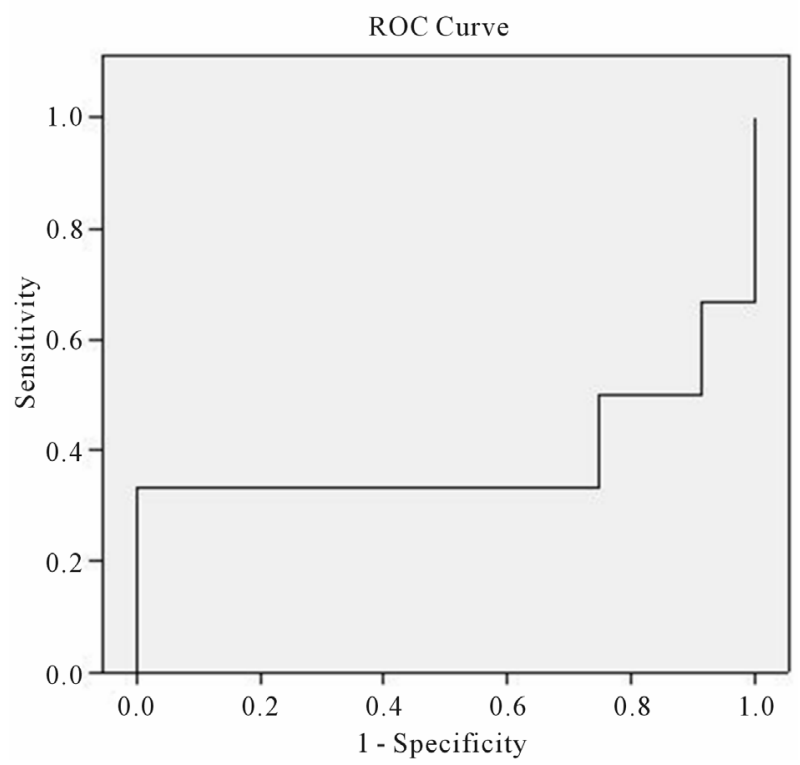

(b)

Graphic 1. (a) ROC curve analysis of cancer patients for ADC values; (b) ROC curve analysis of control group for ADC values.

For H-MRS: Reader 1 found out Cho/Cit and Cho + $\mathrm{Cre} / \mathrm{Cit}$ ratios of cancer patients as $5.36 \pm 5.70,7.63 \pm$ 8.45. These ratios were $5.30 \pm 5.71$ and $7.59 \pm 8.47$ for reader 2. In the control group; Cho/Cit and Cho $+\mathrm{Cre} / \mathrm{Cit}$ ratios were $0.69 \pm 1.11,1.06 \pm 1.42$ for both readers (Table 3, Figure 3). These values were taken as the threshold ratio through all zones of prostate (RA, RT, RP, LA, LT, LP) for being benign. According to Cho/ Cit and Cho + Cre/Cit ratios, cancer patients had significant statistical differences against normal control group $(\mathrm{p}<0.05)$. With regard to biopsy yields $\mathrm{Cho} / \mathrm{Cit}$ and Cho $+\mathrm{Cre} / \mathrm{Cit}$ ratios of both reader's results had significant statistical differences, with $49 \%$ specificity for both ratios, 
Table 3. The results of H-MRS according to each observers (O1: observer 1 and O2: observer 2), cho/ci ratio and Cho + $\mathrm{Cr} / \mathrm{Ci}$ ratio for both observers with respect to biopsy results.

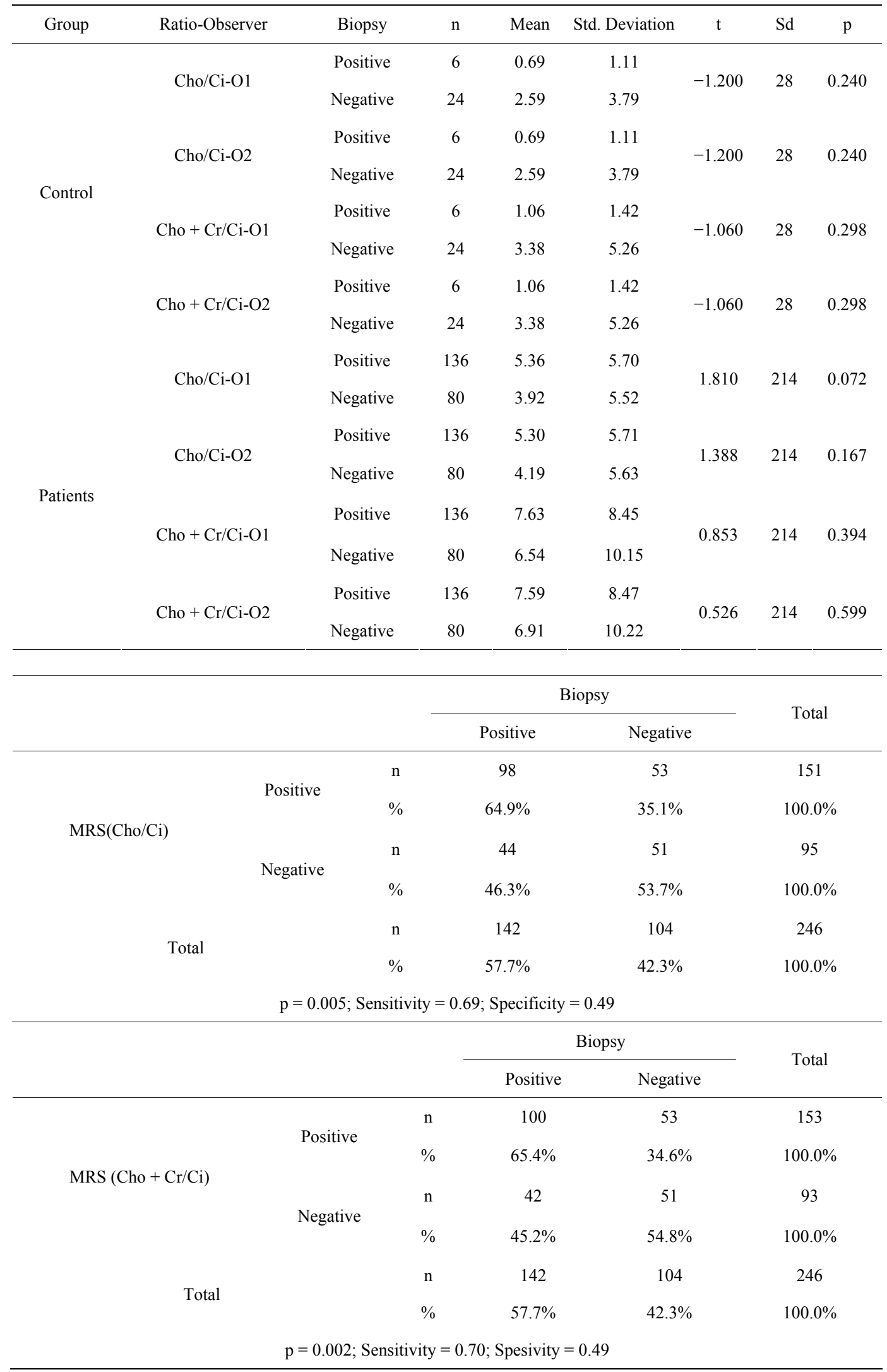




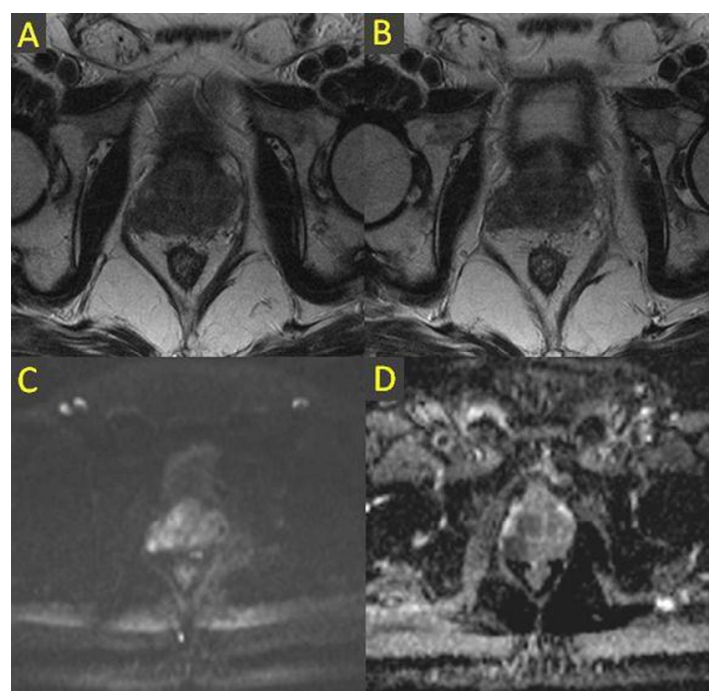

Figure 2. Two consecutive $T 2 W$ images of a patient $(A, B)$ show irregular hypointens area on the right peripheral zone, DWI (C) and ADC mapping (D) depicts restricted diffusion in the left peripheral, transitional and central zones.

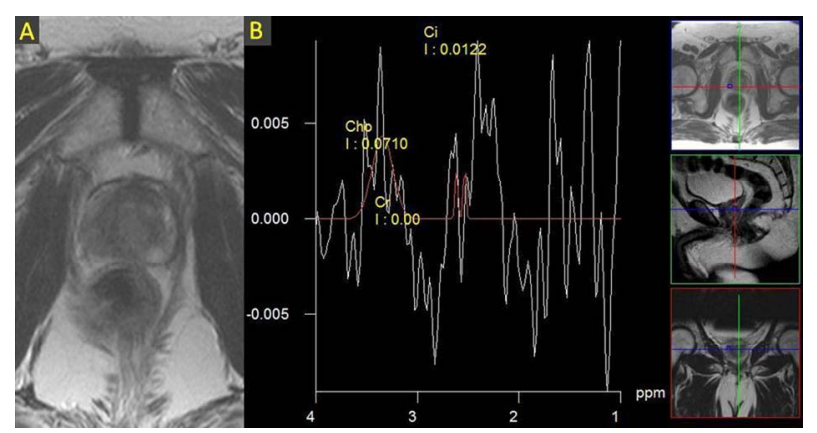

Figure 3. T2W image (A) shows irregular hypoinensity on the right side (in peripheral, transitional and central zones) and H-MRS (B) depicts high $\mathrm{Cho} / \mathrm{Cit}$ ratio in right peripheral zone.

$69 \%$ sensitivity for Cho/Cit and $70 \%$ sensitivity for Cho + Cre/Cit ratio $(\mathrm{p}<0.05)$ (Table 3, Graphic 1(a), Figure 4). With ROC curve analysis; Both readers had 0.99 cut-off value for $\mathrm{Cho} / \mathrm{Cit}$ and 0.98 for $\mathrm{Cho}+\mathrm{Cre} / \mathrm{Cit}$ ratios, $\mathrm{AUC}$ of $\mathrm{Cho} / \mathrm{Cit}: 0.613 \pm 0.04$ for reader $1-$ $0.593 \pm 0.022$ for reader $2, \mathrm{AUC}$ of Cho + Cre/Cit: 0.606 \pm 0.01 for reader $1-0.587 \pm 0.033$ for reader 2 . For both readers; Cut-off value equal or greater than 0.99 for Cho/Cit, 0.98 for Cho + Cre/Cit ratios; regarded $100 \%$ sensitivity and specificity (Graphics 2(a)-(d)). For control group ROC curve analysis; Both readers had the same evaluation: Cut-off value for $\mathrm{Cho} / \mathrm{Cit}$ ratio was equal or more than 0.990 under the AUC: $0.420 \pm 0.111$, regarded $100 \%$ sensitivity and specificity. Cut-off value for Cho + Cre/Cit ratio was equal or more than 0.940, under the AUC; $0.465 \pm 0.105$, indicated $100 \%$ sensitivity and specificity. (Graphics 3(a) and (b)).

When we compare each sequences statistically; T2W

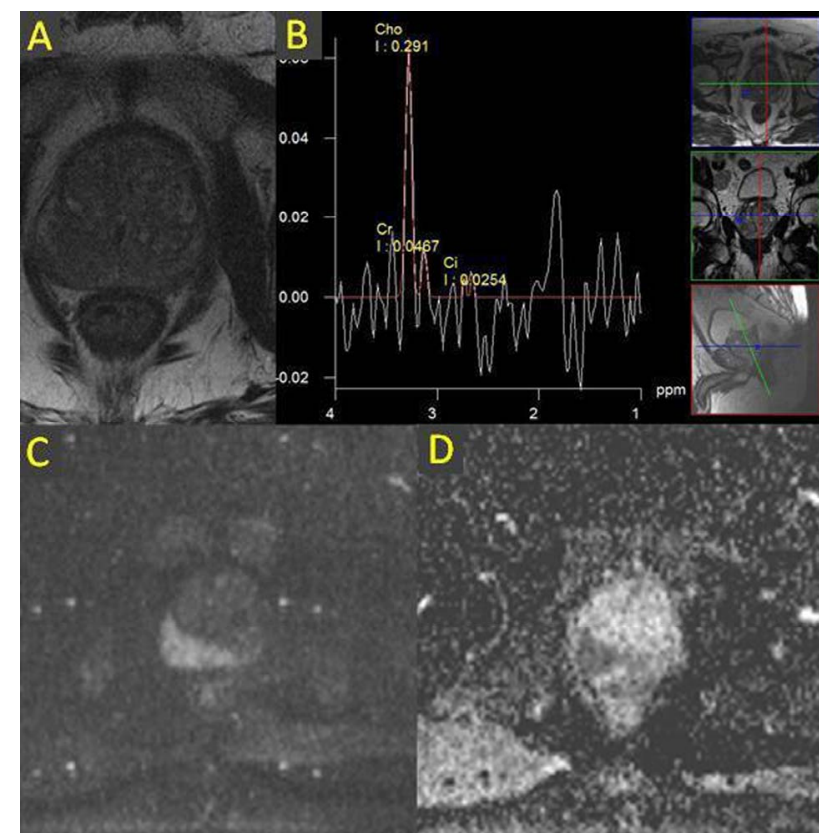

Figure 4. T2W image (A), shows hypointens area both peripheral zones (especially right side). H-MRS (B) depicts high $\mathrm{Cho} / \mathrm{Cit}$ ratio in right peripheral zone. DWI (C) and ADC map (D) reveal prominent restricted diffusion in these zones.

sequence had significant statistical superiority to DWIADC mapping with $50 \%$ sensitivity and $92 \%$ specificity $(\mathrm{p}<0.05)$ (Table 4). H-MRS for both $\mathrm{Cho} / \mathrm{Cit}$ and Cho + Cre/Cit ratios had significant statistical superiority over DWI, had $78 \%$ sensitivity for both ratios- $43 \%$ specificity for $\mathrm{Cho} / \mathrm{Cit}$ and $42 \%$ specificity for $\mathrm{Cho}+$ $\mathrm{Cre} / \mathrm{Cit}$ ratios $(\mathrm{p}>0.05)$ (Table 4). H-MRS for Cho + $\mathrm{Cre} / \mathrm{Cit}$ ratio, regarded statistical superiority over $\mathrm{T} 2 \mathrm{~W}$ sequence with $73 \%$ sensitivity and $42 \%$ specificity $(p<$ 0.05 ) and also superior to T2W sequence in case of Cho/ Cit ratio without any statistical proof by $70 \%$ sensitivity and $42 \%$ specificity $(p>0.05)$. By the combined use of these three sequences; T2W + DWI-ADC map + H-MRS (Cho/Cit and $\mathrm{Cho}+\mathrm{Cre} / \mathrm{Cit}$ ratios) regarded $81 \%$ sensitivity and $66 \%$ specificity, with significant statistical differences to the reference standard, histopathology $(\mathrm{p}<$ $0.05)$.

\section{Discussion}

Screening fundamentals of prostate cancer were based on those patients diagnosed at imaging, tend to present a more favorable stage compared to the diagnosed cases, with a possible decreased rate of specific mortality due to prostate cancer $[1,2,5]$. Magnetic resonance imaging was commonly utilized for tumor staging, clearly demonstrating prostate anatomy and representing focal or diffuse lesions through the prostate [1,4-8]. MR imaging was also widely used for an extensive evaluation of pa- 


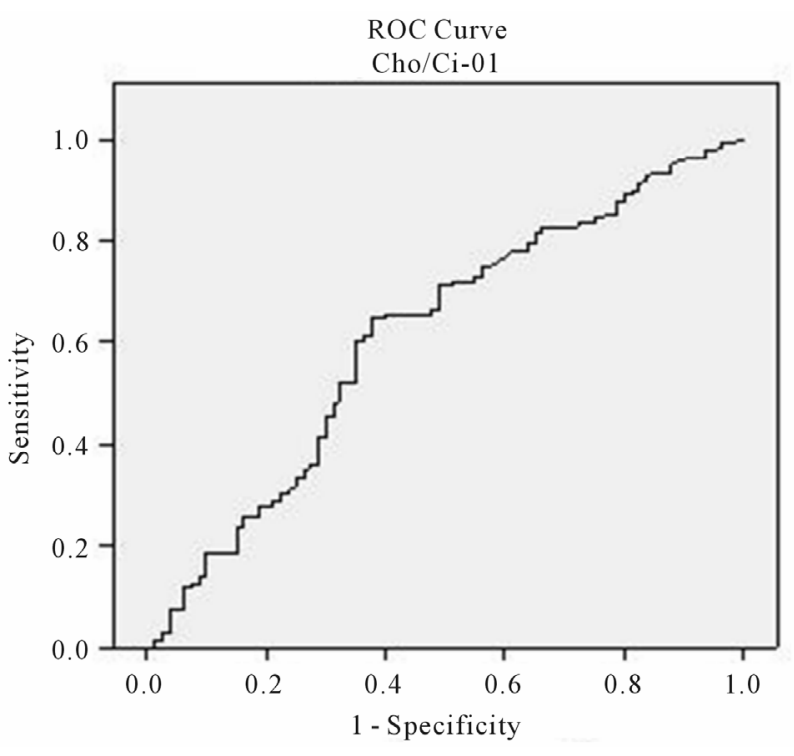

(a)

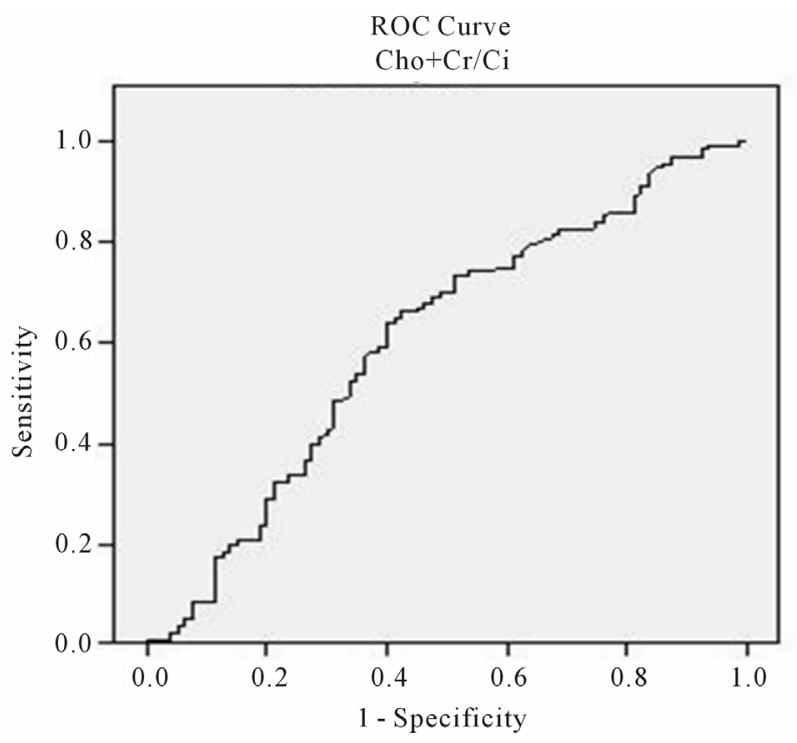

(c)

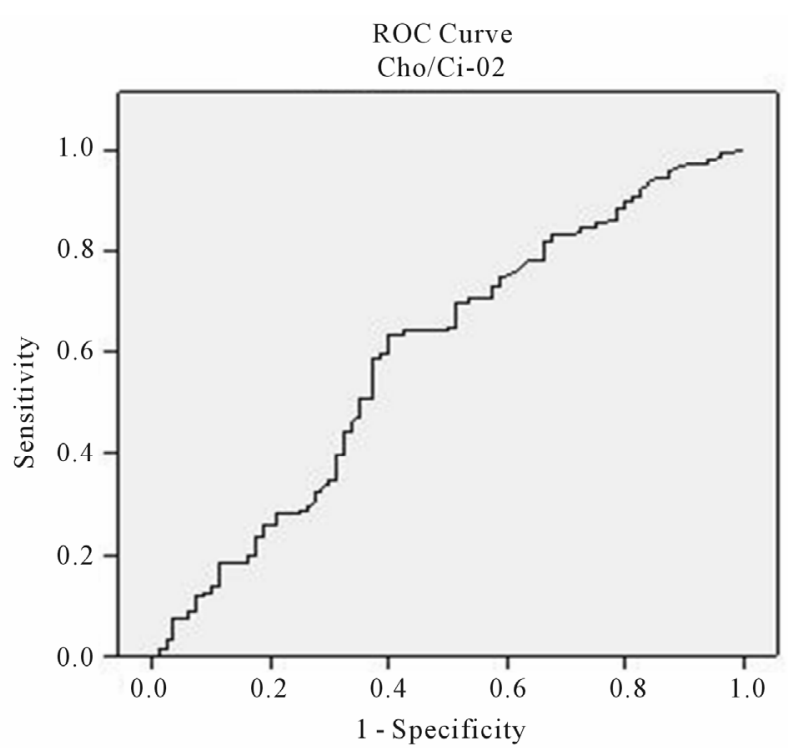

(b)

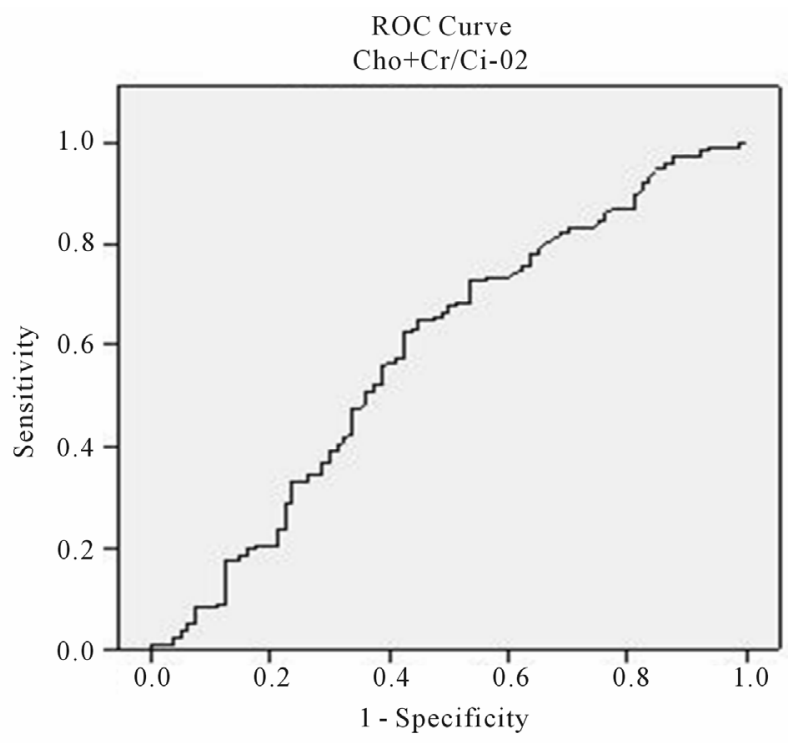

(d)

Graphic 2. (a) ROC curve analysis of MRS (Cho/Ci) ratio in cancer patients for observer 1; (b) ROC curve analysis of MRS $(\mathrm{Cho} / \mathrm{Ci})$ ratio in cancer patients for observer 2; (c) ROC curve analysis of MRS $(\mathrm{Cho}+\mathrm{Cr} / \mathrm{Ci})$ ratio in cancer patients for observer 1; (d) ROC curve analysis of MRS (Cho + Cr/Ci) ratio in cancer patients for observer 2.

tients with prostate cancer, for its capacity of observation of the primary disease and locoregional lymph nodes involvement [1,5-9]. On T1-weighted images, prostate appereance was homogenous with isointense signal, zonal anatomy and intraprostatic diseases were not demonstrated respectively but could be used to detect areas of post-biopsy hemorrhages $[1,4,5,15]$. T2-weighted imaging might regard the cancer with hypointense signal at different prostatic zones but those T2-signal lost areas might also be due to a number of causes; including inflammation, glandular fibrosis and dysplasia, hemorrhage, calcification etc., predicting better sensitivity $(78 \%-89 \%)$ with lower specificity $(40 \%-55 \%)$ in the detection and localization of prostate cancer $[1,4,5,7,10,12,14]$. Additional procedures, such as DWI-ADC mapping and MRSpectroscopy should be applied to achieve a more specific diagnosis and more accurate localization of cancer [1,2,4,7,12,16-21]. Combined T1 and T2-W MR imaging plus H-MRS or H-MRS plus DWI or routine MR imaging plus DWI indicated higher sensitivity $(81 \%-95 \%)$ and specificity $(76 \%-91 \%)$ as seen in the reported articles $[2,7,10,12,14,16,17,20]$.

H-MRS provides information about the metabolite concentrations within a voxel to show the aggressiveness 


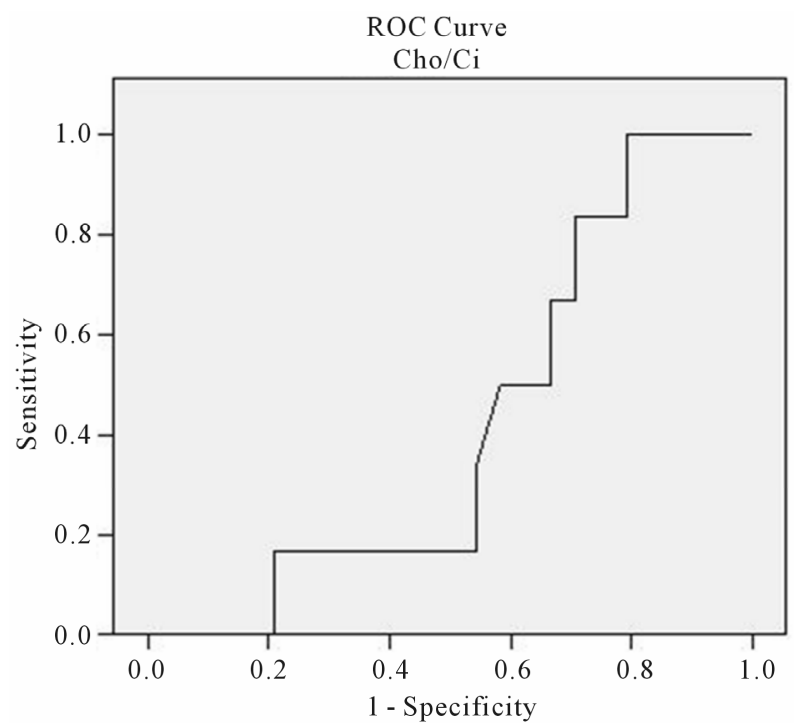

(a)

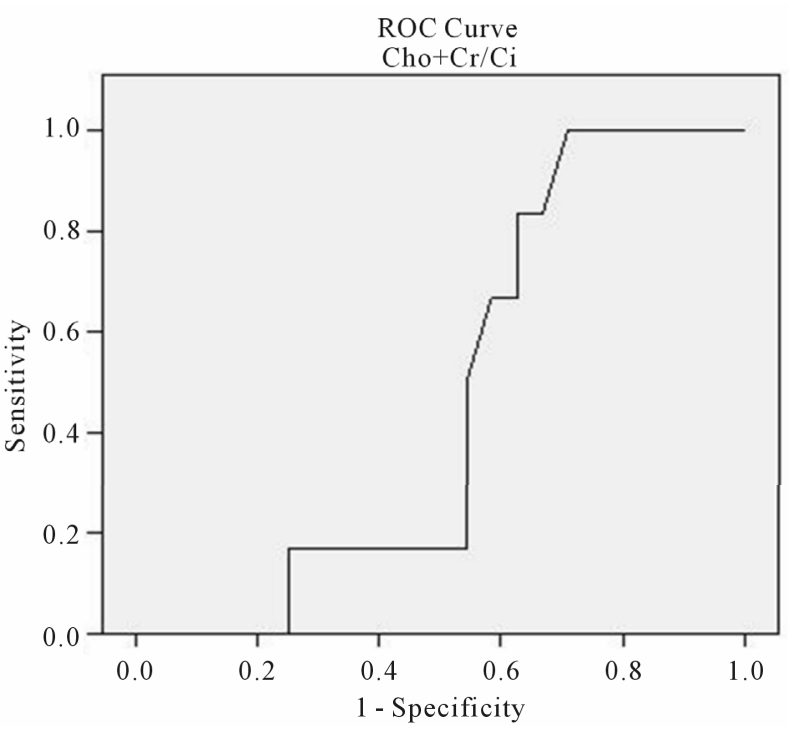

(b)

Graphic 3. (a) ROC curve analysis of MRS (Cho/Ci) ratio in control group; (b) ROC curve analysis of MRS (Cho + $\mathrm{Cr} / \mathrm{Ci}$ ) ratio in control group.

of the cancer, to improve the tumor localization and volume estimation with MRI [1,4,9,15,21,23,24]. DWIADC mapping assesses the Brownian motion of free water in tissues, normal prostate tissue is rich in glandular tissue which has higher water diffusion rates, mostly depicted on ADC mapping, prostate cancer show restricted diffusion with high signal on DWI and low signal on ADC maps $[1,2,4,7,12,16,17]$. DWI is an intrinsically low signal to noise ratio sequence, with noisy images and susceptibility artefacts therefore technique benefits from higher field strengths and surface coils, higher b-values can improve lesion detection [1,7,14,18,31-33].

Concerned with T2W-MR imaging, DWI plus ADC
Table 4. The comparison of MRS (Cho/ $\mathrm{Ci}$ and $\mathrm{Cho}+\mathrm{Cr} / \mathrm{Ci})$ results with $\mathrm{T} 2 \mathrm{~W}$ MRI results and comparison between combination of three sequences to the biopsy results.

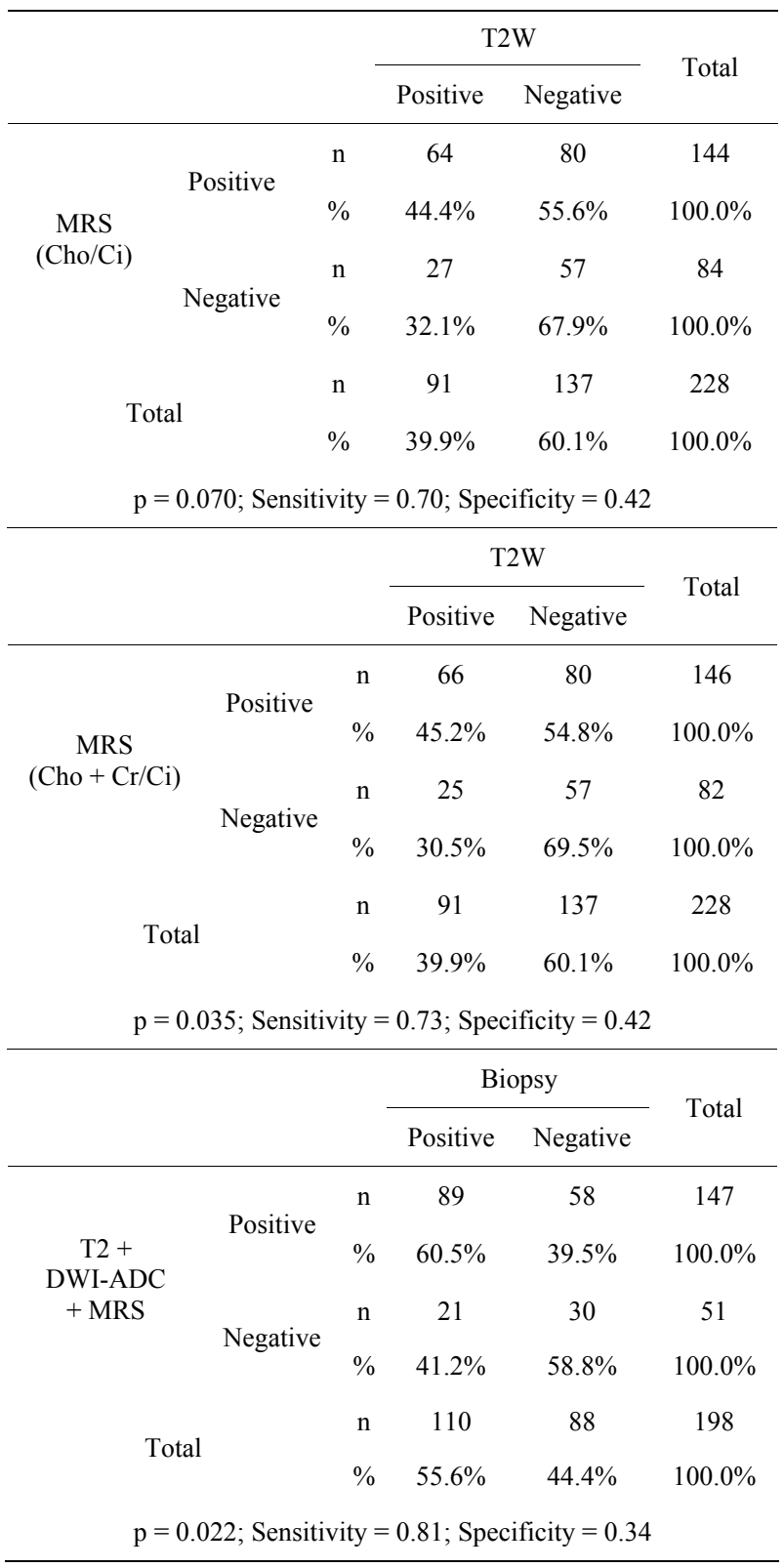

mapping and H-MRS, there were few reports in the literature. Shimofusa et al. [16] demonstrated a study of 37 patients with prostate cancer, 3 readers evaluated the results of $\mathrm{T} 2 \mathrm{~W}$ imaging alone and combined $\mathrm{T} 2 \mathrm{~W}$ and DWI. Sensitivity ranged between $73 \%$ - $86 \%$ for T2W alone and $84 \%$ - $86 \%$ for combined use of $\mathrm{T} 2 \mathrm{~W}$ and DWI images, specificity ranged between $74 \%-83 \%$ for T2W alone and 78\% - 91\% for combined T2W and DWI use which were significantly higher than our results. Haider et al. [12] also compared T2-weighted imaging alone and combined use of T2W and DWI for the localization of prostate cancer in 49 patients, presented 54\% 
sensitivity and 91\% specificity for $\mathrm{T} 2 \mathrm{~W}$ images alone and $81 \%$ sensitivity and $84 \%$ specificity for combined T2W and DWI sequences use, their sensitivities for T2W alone $\mathrm{T} 2 \mathrm{~W}+\mathrm{DWI}$ use and specificity for $\mathrm{T} 2 \mathrm{~W}$ images were higher than our results, specificity for combined use of T2W and DWI images, was almost the same with us. Reinsberg et al. [2] reported increased specificity without a reduction in sensitivity, for combined DWI and 2DHMRS use with comparison to the use of MR-spectroscopy or DWI alone in the voxels containing $70 \%$ or more tumour tissues, with $80 \%$ - $90 \%$ sensitivity and specificity. Our results for $\mathrm{Cho} / \mathrm{Cit}$ ratio and $\mathrm{ADC}$ values were almost the same with lower accuracy.

Mazaheri et al. [7] carried out a research with combined DWI and H-MRS use for detecting prostate cancers, regarded significantly higher $\mathrm{Cho} / \mathrm{Cit}$ ratios and lower ADC values in tumor containing voxels with $85 \%$ $81 \%$ sensitivity and specificity, better than use of DWI or $\mathrm{H}-\mathrm{MRS}$ alone. Our results for $\mathrm{Cho} / \mathrm{Cit}$ ratio and $\mathrm{ADC}$ values were almost the same with lower accuracy. Kumar et al. [31] reported a positive correlation between ADC values and $\mathrm{Cho} / \mathrm{Cit} \mathrm{Cho}+\mathrm{Cre} / \mathrm{Cit}$ ratios in men with elevated PSA levels, reflecting a direct relationship between the reduction of citrate levels and structural changes of prostate tissue associated with malignancy.

Yagc1 et al. [17] declared that DWI+T2W didn't provide significant statistical increase in the diagnostic performance of MRI, with comparison to DWI alone for peripheral zone cancer detection and localization with 1.5 T scanner.

To our belief, this was the first research in the literature analyzing the combined use of T2-weighted imaging, H-MRS and DWI-ADC mapping to detect prostate cancer accurately. We hypothesize that combined information of non-invasive morphologic and functional MR techniques, Multiparametric MR imaging approach rather than use of either sequences alone, could easily improve the detection, localization and staging of prostate cancers. In this research; None of the included sequences alone had adequate sensitivity and specificity for the identification of cancer as $\mathrm{T} 2 \mathrm{~W}$ imaging had $46 \%$ sensitivity and $68 \%$ specificity, DWI and ADC mapping had 29\% sensitivity and $82 \%$ specificity, H-MRS acquisition with Cho/Cit and Cho + Cre/Cit ratios had $49 \%$ specificity for both ratios, $69 \%$ sensitivity for $\mathrm{Cho} / \mathrm{Cit}$ and $70 \%$ sensitivity for Cho $+\mathrm{Cre} / \mathrm{Cit}$ ratio. Even for the use of H-MRS (Most sensitive sequence) and DWI (Most specific one); Was not high enough to diagnose the cancer accurately as three of 10 prostate cancers were missing. In order to improve and validate these results for each sequences; Combination of these MR sequences via multiprametric approach should aid for the depiction and exact localization of prostate cancer. When we add DWI to T2-weighted imaging, the sensitivity raised to $52 \%$ with statistical corre- lation to biopsy without significant improvement in the specificity $(\% 65, \mathrm{p}<0.05)$, combination of $\mathrm{T} 2 \mathrm{~W}$ imaging with H-MRS; A reliable increase for sensitivity was indicated with lower specificity (80\% sensitivity and 32\% specificity) without any statistical proof over histopathology $(p>0.05)$. By combined use of H-MRS and DWI, an increase in sensitivity with lower specificity under statistical approvement to reference standard, was predicted (77\%/43\%, sensitivity/specificity) ( $\mathrm{p}<0.05)$. By Multiparametric MR imaging approach via combined use of all three sequences ; Sensitivity was improved to $81 \%$, a reliable increase for specificity, especially higher than H-MRS use alone were also observed (66\%), multiparametric approach did not acquire more specific results rather than the acquisition of other two sequences alone, with significant statistical correlation to the gold standard $(\mathrm{p}<0.05)$.

We thought that Multiparametric MR imaging approach of these sequences might supply beneficial datas and increase the specificity more than the H-MRS usage alone. By adding T2W and DWI sequences with the application of pelvic-phase array coil; Disadvantages due to lack of endorectal coil and lower spatial resolution should be overcome. An additional MR imaging technique like dynamic contrast enhanced scan might be added to routine prostate MR imaging protocol in order to get higher sensitivity and increase the specificity.

There were several limitations of this research; First, as we didn't have a proper endorectal coil for routine practice, all MRI procedures were acquired by pelvic-phase array coil, this might further cause lower sensitivity and specificity, especially for the DWI-ADC mapping and H-MRS acquisitions. This was the major limitation of this research. Second, relatively a small sampled size and retrospective study design could influence statistical analysis, needed to be confirmed prospectively with large number of cases. Third, small sized tumours within central gland and transitional zone especially $<5$ $\mathrm{mm}$, were easily misdiagnosed by routine $\mathrm{T} 2 \mathrm{~W}$ sequences and $\mathrm{ADC}$ values, Cho $+\mathrm{Cre} / \mathrm{Cit}-\mathrm{Cho} / \mathrm{Cit}$ ratios might not accurately reflect the real tumour situation either and an elevated false-positive rate for benign conditions, such as prostatitis-hemorrhage-glandular hyperplasia-fibrosis etc. might further influence lower specificity of this research especially on $\mathrm{T} 2 \mathrm{~W}$ sequence [4, 14,32,33]. Forth; MRI was performed approximately 4 weeks after prostate biopsy, this factor might cause spectral degradation for H-MRS $[15,23]$ and degrade metabolite signal to noise ratio for MR Spectroscopy, could vary between voxels of the same patient or between them (distance of voxels from coil, magnetic field homogeneity and relaxation times) that might have introduced variability for the outcome. Fifth, DWI had some disadvantages like lower signal to noise ratio, distorted spatial 
resolution, magnetic susceptibility and signal loss which might affect the image quality and the outcome for detection of exact cancer localization $[4,14,19,31,33]$.

\section{Conclusion}

Our study indicated that Multiparametric MR imaging approach by combined use of T2-weighted imaging, diffusion-weighted MR imaging and proton MR-spectroscopy, were more sensitive and more accurate than use of either sequences alone. For exact localization and detection of prostate cancer; if these advanced and newer MR sequences were included in the routine prostate MR protocole; Characterization of prostatic malignancy, discrimination of benign and malignant prostatic tissues, would be significantly improved.

\section{REFERENCES}

[1] B. Turkbey, P. S. Albert, K. Kurdziel and P. L. Choyke, "Imaging Localized Prostate Cancer: Current Approaches and New Developments," AJR, Vol. 192, No. 6, 2009, pp. 1471-1480. doi:10.2214/AJR.09.2527

[2] S. A. Reinsberg, G. S. Payne, S. F. Riches, S. Ashley, J. M. Brewster, V. A. Morgan, et al., "Combined Use of Diffusion-Weighted MRI and H-MR Spectroscopy to Increase Accuracy in Prostate Cancer Detection," AJR, Vol. 188, No. 1, 2007, pp. 91-98. doi:10.2214/AJR.05.2198

[3] C. Obek, P. Louis, F. Civantos and M. S. Soloway, "Comparison of Digital Rectal Examination and Biopsy Results with the radical Prostatectomy Specimen," Journal of Urology, Vol. 161, No. 2, 1999, pp. 494-498. doi:10.1016/S0022-5347(01)61932-3

[4] H. Aydin, V. Kizilgoz, I. Tatar, C. Damar, A. R. Ugan, I. Paker, et al., "Detection of Prostate Cancer with Magnetic Resonance Imaging: Optimization of T1-Weighted, T2Weighted, Dynamic-Enhanced T1-Weighted, Diffusion-Weighted Imaging Apparent Diffusion Coefficient Mapping Sequences and MR Spectroscopy, Correlated With Biopsy and Histopathological Findings," Journal of Computer Assisted Tomography, Vol. 36, No. 1, 2012, pp. 30-45. doi:10.1097/RCT.0b013e31823f6263

[5] E. Farias, H. J. Melo, D. Szejnfeld, C. S. Paiva, N. Abdala, H. O. Arruda, S. M. Goldman, et al., "Magnetic Resonance Spectroscopy Imaging in the Diagnosis of Prostate Cancer: Initial Experience," Radiologia Brasileira, Vol. 42, No. 1, 2009, pp. 1-8.

[6] H. Hricak, "MR Imaging and MR Spectroscopic Imaging in the Pre-Treatment Evaluation of Prostate Cancer," The British Journal of Radiology, Vol. 78, No. 2, 2005, pp. 103-111. doi:10.1259/bjr/11253478

[7] Y. Mazaheri, A. Shukla-Dave, H. Hricak, S. W. Fine, J. Zhang, G. Inurrigarro, et al., "Prostate Cancer: Identification with Combined Diffusion-Weighted MR Imaging and 3D-HMR Spectroscopic Imaging-Correlation with Pathologic Findings," Radiology, Vol. 246, No. 2, 2008, pp. 480-488. doi:10.1148/radiol.2462070368

[8] F. G. Claus, H. Hricak and R. R. Hattery, "Pretreatment
Evaluation of Prostate Cancer: Role of MR Imaging and H-MR Spectroscopy," RadioGraphics, Vol. 24, No. 1, 2004, pp. 167-180.

[9] S. Katz and M. Rosen, "MR Imaging and MR Spectroscopy in Prostate Cancer Management," Radiologic Clinics of North America, Vol. 44, No. 5, 2006, pp. 723-734. doi:10.1016/j.rcl.2006.07.008

[10] A. Wetter, T. A. Engl, D. Nadjamadi, K. Fliessbach, T. Lehnert, J Gurung, et al., "Combined MRI and MR Spectroscopy of the Prostate before Radical Prostatectomy," AJR, Vol. 187, No. 3, 2006, pp. 724-730. doi:10.2214/AJR.05.0642

[11] F. V. Coakley, A. Quayyum and J. Kurhanewicz, "Magnetic Resonance İmaging and Spectroscopic İmaging of Prostate Cancer," Journal of Urology, Vol. 170, No. 6, 2003, pp. 69-75. doi:10.1097/01.ju.0000094958.23276.c4

[12] M. A. Haider, T. H. Van der Kwast, J. Tanguay, A. J. Evans, A. T. Hashmi, G. Lockwood, et al., "Combined T2-Weighted and Diffusion-Weighted MRI for Localization of Prostate Cancer," AJR, Vol. 189, No. 2, 2007, pp. 323-328. doi:10.2214/AJR.07.2211

[13] S. Ikonen, L. Kivisaari, P. Tervahartiala, T. Vehmas, K. Taari and S. Rannikko, "Prostatic MR Imaging: Accuracy in Differentiating Cancer from Other Prostatic Disorders," Acta Radiologica, Vol. 42, No. 4, 2001, pp. 348354.

[14] H. Aydin, B. Hekimoglu and V. Kızılgöz, "A Brief Review for the Combined Use of T2-Weighted MR İmaging and Diffusion Weighted Imaging for Prostate Cancer Diagnosis," AJR, Vol. 200, No. 2, 2012, p. W219. doi:10.2214/AJR.12.9629

[15] J. J. Fütterer, S. W. Heijmink, T. W. Scheenen, J. Veltman, H. J. Huisman, P. Vos, et al., "Prostate Cancer Localization with Dynamic Contrast-Enhanced MR Imaging and Proton MR Spectroscopic Imaging," Radiology, Vol. 241, No. 2, 2006, pp. 449-458. doi:10.1148/radiol.2412051866

[16] R. Shimofusa, H. Fujimoto, H. Akamata, K. Motoori, S. Yamamoto, T. Ueda, et al., "Diffusion-Weighted Imaging of Prostate Cancer," Journal of Computer Assisted Tomography, Vol. 29, No. 2, 2005, pp. 149-153. doi:10.1097/01.rct.0000156396.13522.f2

[17] A. B. Yağci, N. Ozari, Z. Aybek and E. Düzcan, "The Value of Diffusion-Weighted MRI for Prostate Cancer Detection and Localization," Diagnostic and Interventional Radiology, Vol. 17, No. 2, 2011, pp. 130-134.

[18] K. Hosseinzadeh and S. D. Schwarz, "Endorectal Diffusion Weighted Imaging in Prostate Cancer to Differentiate Malignant and Benign Peripheral Zone Tissue," Journal of Magnetic Resonance Imaging, Vol. 20, No. 4, 2004, pp. 654-661. doi:10.1002/jmri.20159

[19] K. C. Kim, K. B. Park, J. J. Han, T. W. Kang and H. M. Lee, "Diffusion-Weighted Imaging of the Prostate at 3T for Differentiation of Malignant and Benign Tissue in Transition and Peripheral Zones: Preliminary Results," Journal of Computer Assisted Tomography, Vol. 31, No. 2, 2007, pp. 449-454. doi:10.1097/01.rct.0000243456.00437.59

[20] J. Kurhanewicz, M. G. Swanson, S. J. Nelson and D. B. 
Vigneron, "Combined Magnetic Resonance Imaging and Spectroscopic Imaging Approach to Molecular Imaging of Prostate Cancer," Journal of Magnetic Resonance Imaging, Vol. 16, No. 4, 2002, pp. 451-463.

doi:10.1002/jmri.10172

[21] J. Kurhanewicz and D. B. Vigneron, "Advances in MR Spectroscopy of the Prostate. Journal of Magnetic Resonance Imaging, Vol. 16, No. 4, 2008, pp. 697-710. doi:10.1016/j.mric.2008.07.005

[22] K. L. Zakian, S. Eberhardt, H. Hricak, A. Shukla-Dave, S. Kleinman, M. Muruganandham, et al., "Transition Zone Prostate Cancer: Metabolic Characteristics at H-MR Spectroscopic Imaging-Initial Results," Radiology, Vol. 229, No. 1, 2003, pp. 241-247. doi:10.1148/radiol.2291021383

[23] D. Pucar, A. Shukla-Dave, H. Hricak, C. S. Moskowitz, K. Kuroiwa, S. Olgac, et al., "Prostate Cancer: Correlation of MR Imaging and MR Spectroscopy with Pathologic Findings after Radiation Therapy-Initial Experience," Radiology, Vol. 236, No., 2005, pp. 545-553. doi:10.1148/radiol.2362040739

[24] U. G. Lisse-Mueller, D. B. Vigneron, H. Hricak, M. G. Swanson, P. R. Carroll, A. Bessette, et al., "Localized Prostate Cancer: Effect of Hormone Deprivation Therapy Measured by Using Combined Three-Dimensional H-MR Spectroscopy and MR Imaging: Clinicopathologic CaseControlled Study," Radiology, Vol. 221, No. 2, 2001, pp. 380-390. doi:10.1148/radiol.2211001582

[25] A. C. Westphalen, F. V. Coakley, A. Quayyum, M. G. Swanson, J. P. Simko, Y. Lu, et al., "Peripheral Zone prostate Cancer: Accuracy of Different Interpretative Approaches with MR and MR Spectroscopic Imaging," Radiology, Vol. 246, No. 1, 2008, pp. 177-184. doi:10.1148/radiol.2453062042

[26] H. Aydin, N. A. Oktay, V. Kizilgoz, E. Altin, I. G. Tatar and B. Hekimoglu, "Value of Proton-MR-Spectroscopy in the Diagnosis of Temporal Lobe Epilepsy; Correlation of Metabolite Alterations with Electroencephalography," Iranian Journal of Radiology, Vol. 9, No. 1, 2012, pp. 1-11. doi:10.5812/iranjradiol.6686

[27] H. Aydin, V. Kizilgoz, I. Tatar, C. Damar, H. Guzel, B. Hekimoglu, et al., "The Role of Proton MR Spectroscopy and Apparent Diffusion Coefficient Values in the Diagnosis of Malignant Thyroid Nodules: Preliminary Results," Clinical Imaging, Vol. 36, No. 4, 2011, pp. 323-333.

[28] H. Aydin, S. Sipahioğlu, N. A. Oktay, E. Altin, V. Kizilgöz and B. Hekimoglu, "The Value of Proton-MRSpectroscopy in the Differentiation of Brain Tumours from Non-Neoplastic Brain Lesions," JBR-BTR, Vol. 94, No. 1, 2011, pp. 1-10.

[29] G. L. Lu-Yao and S. L. Yao, "Population-Based Study of Long-Term Survival in Patients with Clinically Localized Prostate Cancer," Lancet, Vol. 349, No. 9056, 1997, pp. 906-910. doi:10.1016/S0140-6736(96)09380-4

[30] J. R. Landis and G. G. Koch, "The Measurement of Observer Agreement for Categorical Data," Biometrics, Vol. 33, No. 1, 1977, pp. 159-174. doi:10.2307/2529310

[31] V. Kumar, N. R. Jagannathan, R. Kumar, S. C. Das, L. Jindal, S. Thulkar, et al., "Correlation between Metabolite ratios and ADC Values of Prostate in Men with Increased PSA Level," Magnetic Resonance Imaging, Vol. 24, No. 5, 2006, pp. 541-548. doi:10.1016/j.mri.2006.01.001

[32] H. K. Lim, J. K. Kim, K. A. Kim and K. Cho, "Prostate Cancer: ADC with T2 Weighted Images for DetectionA Multireader Study," Radiology, Vol. 250, No. 3, 2009, pp. 145-151. doi:10.1148/radiol.2501080207

[33] H. Aydin, "A New Approach for Prostate Cancer Diagnosis: Perfusion and Diffusion Measurements," AJR, 2012 , in press. 